\title{
Regulation of cell distancing in peri-plaque glial nets by Plexin-B1 affects glial activation and amyloid compaction in Alzheimer's disease
}

Roland Friedel ( $\square$ roland.friedel@mssm.edu)

Icahn School of Medicine at Mount Sinai https://orcid.org/0000-0002-7513-3604

\section{Yong Huang}

Icahn School of Medicine at Mount Sinai https://orcid.org/0000-0001-8095-4215

\section{Minghui Wang}

Department of Genetics and Genomic Sciences, Icahn School of Medicine at Mount Sinai https://orcid.org/0000-0001-9171-4962

\section{Shalaka Wahane}

Icahn School of Medicine at Mount Sinai https://orcid.org/0000-0002-3795-6407

\section{Mitzy Ríos de Anda}

Icahn School of Medicine at Mount Sinai

\section{Lap Ho}

Icahn School of Medicine at Mount Sinai

Yuhuan Li

Icahn School of Medicine at Mount Sinai

\section{Sangjo Kang}

Icahn School of Medicine at Mount Sinai

\section{Ryan Neff}

Icahn School of Medicine at Mount Sinai

Ana Kostic

Icahn School of Medicine at Mount Sinai

Joseph Buxbaum

Mount Sinai School of Medicine

John Crary

Icahn School of Medicine at Mount Sinai

Bin Zhang

Icahn School of Medicine at Mount Sinai

Hongyan Zou

Icahn School of Medicine at Mount Sinai 
Keywords: glial cells, astrocytes, amyloid deposits

Posted Date: October 28th, 2021

DOI: https://doi.org/10.21203/rs.3.rs-967160/v1

License: (c) (1) This work is licensed under a Creative Commons Attribution 4.0 International License. Read Full License 


\section{Regulation of cell distancing in peri-plaque glial nets by Plexin-B1 affects glial activation and amyloid compaction in Alzheimer's disease}

Yong Huang 1,2,3, Minghui Wang ${ }^{2,3}$, Shalaka Wahane ${ }^{1}$, Mitzy Ríos de Anda ${ }^{4}$, Lap Ho ${ }^{2,3}$, Yuhuan Li ${ }^{1}$, Sangjo Kang ${ }^{1}$, Ryan Neff ${ }^{2,3}$, Ana Kostic ${ }^{4}$, Joseph Buxbaum ${ }^{4}$, John F. Crary ${ }^{1,5-8}$, Bin Zhang ${ }^{2,3,9}{ }^{*}$, Hongyan Zou $^{1,10^{*}}$, Roland H. Friedel ${ }^{1,10 *}$

${ }^{1}$ Nash Family Department of Neuroscience, Friedman Brain Institute, Icahn School of Medicine at Mount Sinai, New York, NY, USA

2 Department of Genetics and Genomic Sciences, Icahn Institute of Genomics and Multiscale Biology, Icahn School of Medicine at Mount Sinai, New York, NY, USA

${ }^{3}$ Mount Sinai Center for Transformative Disease Modeling, Icahn School of Medicine at Mount Sinai, New York, NY, USA

${ }^{4}$ Seaver Autism Center, Department of Psychiatry, Icahn School of Medicine at Mount Sinai, New York, NY, USA

${ }^{5}$ Department of Pathology, Icahn School of Medicine at Mount Sinai, New York, NY, USA

${ }^{6}$ Department of Artificial Intelligence and Human Health, Icahn School of Medicine at Mount Sinai, New York, NY, USA

${ }^{7}$ Neuropathology Brain Bank \& Research Core, Icahn School of Medicine at Mount Sinai, New York, NY, USA

${ }^{8}$ Ronald M. Loeb Center for Alzheimer's Disease, Icahn School of Medicine at Mount Sinai, New York, NY, USA

${ }^{9}$ Department of Pharmacological Sciences, Icahn School of Medicine at Mount Sinai, New York, NY, USA

${ }^{10}$ Department of Neurosurgery, Icahn School of Medicine at Mount Sinai, New York, NY, USA

* Corresponding authors:

Bin Zhang, bin.zhang@mssm.edu

Hongyan Zou, hongyan.zou@mssm.edu

Roland H. Friedel, roland.friedel@mssm.edu 


\section{ABSTRACT}

Communication between glial cells has a profound effect on the pathophysiology of Alzheimer's disease (AD), but the underlying mechanisms remain unclear. Here, we reveal a role of reactive astrocytes in enforcing cell distancing in the glial nets surrounding amyloid plaques, which restricts microglial coverage of $\mathrm{A} \beta$, a prerequisite to detect and engulf amyloid deposits. This process is mediated through guidance receptor Plexin-B1, which we identified as a key network regulator of late-onset AD. We show that PlexinB1 is robustly upregulated in plaque-associated astrocytes in a corona-like pattern, and its expression levels correlate with plaque burden and disease severity in AD patients. In APP/PS1 mice, an amyloidogenic model of AD, removing Plexin-B1 led to smaller peri-plaque glial nets with relaxed cell distancing and enhanced glial coverage of $\mathrm{A} \beta$ plaques, as well as transcriptional changes in both reactive astrocytes and disease-associated microglia that are linked to glial activation and amyloid clearance. Furthermore, amyloid plaque burden was lowered, together with a shift towards dense-core plaques and reduced neuritic dystrophy. Our data thus support a role of Plexin-B1 in controlling glial net structure by imposing cell distancing, leading to poor glial coverage of $\mathrm{A} \beta$, reduced amyloid clearance and compaction. Relaxing cell distancing by targeting guidance receptors may present an alternative strategy to alleviate neuroinflammation in $\mathrm{AD}$ by improving glial coverage of $\mathrm{A} \beta$ amyloid and plaque compaction. 


\section{INTRODUCTION}

Alzheimer's disease (AD) remains a major medical challenge with no effective treatments at hand (Long and Holtzman, 2019). The cognitive decline and memory loss in AD patients are caused by neurodegeneration linked to deposition of extracellular amyloid $\beta(\mathrm{A} \beta)$ and intracellular neurofibrillary tangles of phospho-Tau protein (Gandy and DeKosky, 2013). Accumulation of amyloid plaques is a pathological hallmark of $\mathrm{AD}$ (Gandy and Ehrlich, 2021), but the governing factors of plaque development -i.e., A $\beta$ deposition, clearance, and compaction - are not fully understood.

Glial communication is essential for maintaining brain homeostasis and injury responses upon pathological stimuli (Matejuk and Ransohoff, 2020). Recent advances have highlighted the importance of microglia in engulfing and clearing $\mathrm{A} \beta$ in $\mathrm{AD}$ brains (Leng and Edison, 2021). Microglial phagocytosis of amyloid is also required to form compact dense-core plaques, which can limit the exposure of $A \beta$ amyloid to nearby healthy neurons (Yuan et al., 2016). In addition, microglial coverage of amyloid plaques may also serve as a barrier to prevent exposure of neurotoxic protofibrillar A $\beta$ hotspots to neurites (Condello et al., 2015). However, what controls microglial coverage of $\mathrm{A} \beta$, a prerequisite to detect and engulf amyloid material, is not well-defined.

Reactive astrocytes respond to CNS pathology by upregulating GFAP and extending hypertrophic arborized processes (Smit et al., 2021). In AD, amyloid plaques are surrounded by islands of reactive astrocytes and activated microglia, forming a 'reactive glial nets' (Bouvier et al., 2016), wherein glial cells closely interact with one another and with $\mathrm{A} \beta$. Indeed, it has been shown that microglia-derived factors, such as IL-1 $\alpha, \mathrm{TNF} \alpha$, and $\mathrm{C} 1 \mathrm{q}$, can induce astrocytes into an inflammatory phenotype (Liddelow et al., 2017). However, less is understood of how cellular organization and physical contacts with amyloid plaques are governed in reactive glial nets, which has important ramifications for glial cell activation and plaque 
development.

Earlier research on reactive astrocytes in $\mathrm{AD}$ has mainly focused on roles of ApoE, GFAP, vimentin, connexins, and STAT3 (Smit et al., 2021), but the knowledge of the gene program operating in plaqueassociated astrocytes is incomplete. Multiscale network analysis of large-scale genetics and functional genomics data in AD provides a new framework to nominate novel molecular players that influence disease mechanisms (Zhang et al., 2013). Application of this framework to the multi-Omics data in the Mount Sinai Brain Bank AD cohort (MSBB-AD) (Wang et al., 2018) has identified Plexin-B1 (gene symbol PLXNB1) as a key network regulators of late-onset AD (Wang et al., 2020).

Plexin-B1 is a member of the plexin family of axon guidance receptors (Tran et al., 2007). Plexins and the cognate semaphorin ligands regulate cell-cell communication in a variety of contexts, including development, adult physiology, and cancer (Gurrapu and Tamagnone, 2016; Hota and Buck, 2012; Jongbloets and Pasterkamp, 2014; Koropouli and Kolodkin, 2014). The domain structure of plexins is evolutionarily remarkably conserved, including the ring-shaped extracellular domain and the intracellular Ras-GAP (GTPase activating protein) domain, supporting a regulatory role in cytoskeletal dynamics and cell adhesion complexes via small GTPases (Junqueira Alves et al., 2021). Plexin-B1 binds to class 4 semaphorins, a family with six members expressed in diverse cell types (Zhou et al., 2008). Plexin-B1 knockout $(\mathrm{KO})$ mice are viable and fertile, with no overt developmental defects in the brain (Deng et al., 2007; Fazzari et al., 2007). Subsequent studies have shown that Plexin-B1 mediates several aspects of adult physiology, such as immune system activation and bone homeostasis (Negishi-Koga et al., 2011; Okuno et al., 2010; Takamatsu et al., 2010).

In the adult brain, Plexin-B1 is primarily expressed in astrocytes (Zhang et al., 2016). Besides our own investigations, recent studies by other teams have also independently implicated Plexin-B1 in AD: First, 
PLXNB1 was identified as a key node in a gene module highly associated with cognitive decline in AD patients of the ROS-MAP cohort (Mostafavi et al., 2018). Second, proteomics studies revealed a positive correlation of Plexin-B1 protein levels in AD patients with amyloid plaque load and tau neurofibrillary tangle density (Yu et al., 2018). Third, single nucleus RNA-sequencing identified PLXNBI as one of the top induced genes in astrocytes in early stages of AD (Mathys et al., 2019). While these expression data all suggest a role of Plexin-B1 in mediating astrocytic responses to AD pathology, in vivo functional data are lacking.

We therefore set out to identify the functional significance of Plexin-B1 in AD. We show that Plexin-B1 is induced in peri-plaque glial nets in both human and mouse AD brains. Deletion of Plexin-B1 in the APP/PS1 amyloidogenic mouse model of AD drastically altered the glial net structure, with smaller but more compact glial nets, and a higher astrocytic and microglial coverage of amyloid plaques. Absence of Plexin-B1 also induced transcriptional changes in both reactive astrocytes and disease-associated microglia (DAMs), as well as an overall protective phenotype in AD brains, including lowered plaque burden, a shift towards dense-core plaques type, and reduced neuritic dystrophy. Together, our results unveil a role of reactive astrocytes in controlling the structure of glial nets in $\mathrm{AD}$ by imposing cell distancing via the guidance receptor Plexin-B1, which limits glial coverage of amyloid aggregates, thereby increasing neurotoxicity and neuroinflammation. 


\section{RESULTS}

\section{Plexin-B1 is a key network regulator and its expression is correlated with plaque burden}

To uncover new molecular players underlying AD pathophysiology, we conducted a multiscale gene network analysis of the transcriptomic and genomic data from human healthy controls and AD patients in the Mount Sinai Brain Bank cohort (Wang et al., 2018; Wang et al., 2021). We identified Plexin-B1 (gene symbol $P L X N B 1)$ as a hub gene in a coregulated subnetwork underlying late-onset $\mathrm{AD}$ (Fig. 1A). Gene enrichment analysis revealed that the PLXNBI centered network is significantly enriched for astrocytespecific genes and genes upregulated in $\mathrm{AD}$ (Fig. 1B), supporting a potential role of Plexin-B1 in regulating astrocyte reactivity in $\mathrm{AD}$. Concordantly, independent cell type-specific RNA-seq data from both human and mouse brains confirmed that $P L X N B 1$ is predominantly expressed in astrocytes (Fig. S1A). Enrichment analysis also showed that $P L X N B 1$ network genes are significantly enriched for the gene ontology (GO) term "Regulation of small GTPase mediated signal transduction" (Fig. 1B), consistent with plexin signaling through Ras/Rap to control cytoskeleton and cell adhesion dynamics (Bos, 2018; Hota and Buck, 2012). Transcriptomic data from both the superior temporal gyrus and the parahippocampal gyrus, two highly affected areas in AD patients, demonstrated a positive correlation of $P L X N B 1$ expression levels with amyloid plaque burden (Fig. 1C).

\section{Plexin-B1 protein is upregulated in plaque-associated astrocytes in glial nets}

To localize Plexin-B1 protein expression, we performed immunohistochemistry (IHC) on post-mortem brain specimens from AD patients. Strikingly, we detected a corona-like expression pattern of Plexin-B1, reminiscent of the reactive glial nets surrounding plaques in AD brains (Bouvier et al., 2016) (Fig. 1D; Fig. S1B). In cortical areas devoid of amyloid plaques, baseline expression of Plexin-B1 was low (Fig. S1B). Co-immunostaining showed an overlap of Plexin-B1 and GFAP in the glial net (Fig. S1B), consistent with the transcriptomic data that $P L X N B 1$ is predominantly expressed in astrocytes. We extended IHC analyses 
to a cohort of 11 patients spanning a spectrum of disease severity, including 3 individuals without clinical symptoms, and we detected a positive correlation of Plexin-B1 expression scores with the neuropathological ABC scores for amyloid plaques, neurofibrillary tangles, and neuritic plaques (Hyman et al., 2012; Montine et al., 2012) (Fig. 1E).

\section{$P L X N B 1$ is most strongly induced in AD molecular subtypes associated with A $\beta$ pathways}

Our recent transcriptomic analysis of human AD brains revealed five molecular subtypes including typical $(\mathrm{C} 1, \mathrm{C} 2)$, intermediate $(\mathrm{B} 1, \mathrm{~B} 2)$, and atypical (A), that are characterized by different combinations of dysregulated pathways (Neff et al., 2021). We examined PLXNB1 expression in the 5 AD molecular subtypes and found that PLXNB1 is most highly upregulated in the typical AD subtypes C1 and C2 (Fig. 1F), which are closely associated with activation of $A \beta$ related pathways (Neff et al., 2021). Together, our transcriptomic and histological analyses converge on a potential role of Plexin-B1 in astrocyte reactivity in response to amyloid formation in AD brains.

\section{Plexin-B1 controls cell distancing of plaque-associated astrocytes and microglia in glial nets}

To understand the function of Plexin-B1 in AD pathophysiology, we bred a Plxnb1 knockout (PB1-KO) allele (Friedel et al., 2005) into APP/PS1 transgenic mice, an amyloidogenic mouse model of AD (Jankowsky et al., 2004) (Fig. S1C). As specific antibodies against mouse Plexin-B1 were not available to us for IHC (we had tested four commercial antibodies, none demonstrated specificity (Daviaud et al., 2016)), we took advantage of the lacZ reporter contained in the PB1-KO allele to monitor Plxnb1 expression. XGal staining of brain sections from 6-month-old APP/PS1 Plxnb1 $1^{+/-}$mice showed a marked upregulation of LacZ/Plxnb1 that was restricted to peri-plaque areas, whereas in cortical areas devoid of plaques, expression levels were low (Fig. S1D). Hence, Plexin-B1 is robustly upregulated in peri-plaque glial nets in both human and mouse AD brains. 
We next examined the impact of Plexin-B1 ablation on astrocyte reactivity around amyloid plaques. We observed striking differences in astrocyte mobilization and glial net structures in $A P P / P S 1 \mathrm{~PB} 1-\mathrm{KO}$ mice as compared to $A P P / P S 1$ littermates. Specifically, in the cortex of 6-month-old $A P P / P S 1$ mice, amyloid plaques were surrounded by numerous reactive astrocytes with GFAPhigh hypertrophic processes, and they collectively formed ringed glial nets, extending about two-cell layers wide from the plaque. By contrast, in APP/PS1 PB1-KO mice at matching age and cortical area, a markedly decreased number of reactive astrocytes (a reduction of over 50\%) was present around plaques, forming glial nets only about one cell layer deep (Fig. 2A-C; Fig. S2). Furthermore, the cellular spacing of plaque-associated astrocytes appeared reduced in $\mathrm{PB} 1-\mathrm{KO}$, with glial cells crowding in smaller glial nets, with astrocytic processes polarized towards the plaque and displaying more contact with amyloid material and higher plaque coverage (Fig. 2B, C). These phenotypes signify that Plexin-B1 functions to enforce cell distancing in glial nets, leading to a larger but looser peri-plaque glial nets; removing Plexin-B1 resulted in smaller but more compact glial nets with higher plaque coverage by astrocytic processes.

Since glial nets surrounding amyloid plaques consist of both reactive astrocytes and activated microglia (Bouvier et al., 2016), we wondered whether the changes in the recruitment and cellular spacing of plaqueassociated astrocytes in PB1-KO may also affect microglia organization in reactive glial nets. Indeed, IHC

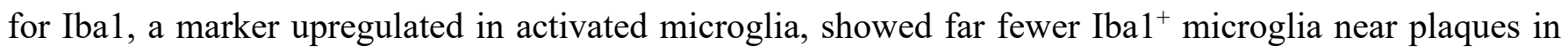
APP/PS1 PB1-KO mice than in APP/PS1 littermates (Fig. 2B, D). As for reactive astrocytes, cellular spacing of plaque-associated microglia was also reduced in PB1 KO, shown by increased microglial density and higher amyloid coverage by microglial processes in the glial nets (Fig. 2D). Hence, Plexin-B1 induction in reactive astrocytes not only exerts cell-autonomous effects on astrocytes, but also affects microglia mobilization and access to $\mathrm{A} \beta$ deposits.

In the absence of $\mathrm{AD}$ pathology, Plexin-B1 $\mathrm{KO}$ alone did not appear to affect the homeostatic astrocyte and 
microglia populations, as GFAP or Iba1 expression in the cortex was comparable between PB1-KO and wildtype (WT) genotypes (Fig. S3A, B). Likewise, in the APP/PS1 background, in cortical areas distant

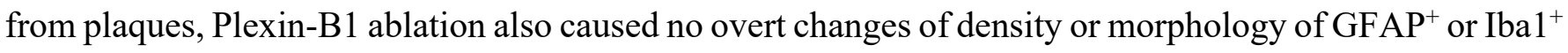
cells (Fig. S3C). Together, these observations demonstrate that Plexin-B1 deletion affects predominantly reactivity of plaque-associated glial cells in response to AD pathology (Fig. 2E).

\section{Plexin-B1 ablation results in transcriptional changes linked to glial function for amyloid clearance}

To gain a global perspective of the impact of Plexin-B1 KO on gene expression changes in AD-affected brains, we performed RNA-sequencing (RNA-seq) on prefrontal cortical tissue from APP/PS1 mice with or without $\mathrm{PB} 1-\mathrm{KO}$ at 3-4 month of age, a timeframe when $\mathrm{A} \beta$ amyloid is being actively deposited, but plaques have not yet fully developed (Fig. S4A). We identified 2,723 differentially expressed genes (DEGs, $P<0.05$ ) between $A P P / P S 1$ PB1-KO and APP/PS1 samples (Fig. S4B). Notably, the upregulated DEGs were enriched for gene ontologies (GO) related to nervous system development (e.g., cerebral cortex neuron differentiation, sympathetic nervous system development, ganglion development), as well as GOs such as cytoskeleton of presynaptic active zone and transmembrane phosphatase activity (Fig. S4C). Downregulated DEGs were enriched for GO terms associated with tissue inflammation (e.g., collagen fibril organization, TNF-activated receptor activity, CD95 death-induced signaling complex, phagolysosome), as well as detection of mechanical stimuli, and protein deacetylase activity (Fig. S4C). Hence, tissue-level transcriptomics supports an overall protective effect of Plexin-B1 KO, with augmented neural repair and attenuated neuroinflammation and cell death.

To dissect cell type-specific gene expression changes in dependence of Plexin-B1 in AD brains, we performed single cell (sc) RNA-seq on prefrontal cortical tissue from APP/PS1 mice with or without PlexinB1 KO at 6 months of age, using the 10X Genomics platform (Fig. S5A, B). After applying quality control filters, we obtained data from a total of 27,286 cells from four genotypes - WT, PB1-KO, APP/PS1, 
APP/PS1 PB1-KO - which were partitioned into 10 distinct cell clusters based on marker gene expression (Fig. 3A; Fig. S5C, D). Microglia occupied the largest cluster (43\%), followed by endothelial cells (22.6\%), astrocytes $(16.6 \%)$, and other cell types with smaller shares, i.e., fibroblasts/smooth muscle cells, macrophages, oligodendrocytes, neurons, pericytes, and ependymal cells (Fig. S5E). The scRNA-seq data confirmed that Plxnbl expression was largely restricted to astrocyte cluster, and it was efficiently ablated in the PB1-KO genotype (Fig. 3B).

Further subclustering analysis of astrocytes identified four subclusters (sc-0 to sc-3) with discrete transcriptional states (Fig. 3C). Subcluster sc-0 represented homeostatic astrocytes with high expression of genes for gap junction assembly, but low expression of genes related to chemokine binding (Fig. S6A). Sc1 showed the strongest association with reactive astrocytes, based on high scores for the gene signature defined for A1-type reactive astrocytes (Liddelow et al., 2017) (Fig. 3D). Notably, A2-type and panreactive (shared by both $\mathrm{A} 1$ and $\mathrm{A} 2$ reactive astrocytes) gene signatures were also enriched in sc-1 astrocytes, albeit with lower scores. Indeed, vimentin, an intermediate filament gene and a marker of panreactive astrocytes, was highly expressed in sc-1 astrocytes (Fig. S6B). Interestingly, Tyrobp and Trem2, two well-known markers of disease-associated microglia (DAM) (Keren-Shaul et al., 2017), but also upregulated in astrocytes in AD conditions (Ceyzériat et al., 2018), were also robustly upregulated in sc-1 astrocytes (Fig. 3E). The much smaller sc-2 specialized in lipid metabolism, while sc-3 may represent a small transitional population with high expression of Gfap and Tyrobp, and genes involved in phagocytosis (Fig. S6A). Plxnb1 mRNA was detected in all astrocyte subclusters, but sc-3 had the highest expression of Plxnb1 (Fig. 3E).

We observed the following features when comparing astrocyte subpopulations in different genotype conditions (Fig. 3F): First, sc-1 reactive astrocytes were greatly expanded in APP/PS1 relative to WT (57\% vs. $12 \%$ ), and correspondingly, sc-0 homeostatic astrocytes showed a shrinkage in APP/PS1 compared to 
WT ( $38 \%$ vs. $78 \%$ ), as did sc-2 astrocytes (2\% vs. $6 \%$ ). Second, PB1-KO caused a marked shift in the representation of the four astrocyte subclusters in $A P P / P S 1$ background, with sc-1 reactive astrocytes reduced to $23 \%$ in $A P P / P S 1 \mathrm{~PB} 1-\mathrm{KO}$ as compared to $57 \%$ in $A P P / P S 1$, while the shares of sc-0 homeostatic astrocytes (66\%) and sc-2 astrocytes (6\%) also returned closer to levels in WT. This agrees with our IF data showing smaller glial nets with fewer reactive astrocytes around plaques in APP/PS1 PB1-KO vs. APP/PS1 brains. Third, PB1-KO alone, without AD transgenic background, resulted in a small expansion of sc-1 reactive astrocytes $(22 \%)$ as compared to WT $(12 \%)$. Together, our transcriptomic data on single cell level provided molecular evidence in support of an influence of Plexin-B1 ablation on the reactivity of astrocytes in response to amyloid plaques.

In addition to reducing the proportion of reactive astrocytes, Plexin-B1 deletion also affected the gene expression profiles of reactive astrocytes. We identified 541 DEGs in sc-1 reactive astrocytes between APP/PS1 PB1-KO and APP/PS1 (>1.25-fold change, $P<0.05)$, with majority upregulated (501) and only 40 downregulated (Fig. 3G; Table S1). Notably, Apoe, a lipid metabolism gene and a risk factor for AD (Long and Holtzman, 2019), was among the top upregulated genes in sc-1 reactive astrocytes as a result of PB1-KO. We corroborated this finding by measuring ApoE protein secretion from cultured primary astrocytes by ELISA, which showed a significantly higher ApoE secretion from PB1-KO astrocytes than WT counterparts at baseline conditions (Fig. S7). Stimulation with A $\beta$ enhanced ApoE secretion from WT astrocytes, but did not further enhance the already high levels of ApoE secretion by PB1-KO astrocytes (Fig. S7).

In further support of an improved functional state of reactive astrocytes regarding amyloid metabolism in Plexin-B1 KO condition, gene ontology analysis showed that the upregulated DEGs in sc-1 reactive astrocytes between $A P P / P S 1 \mathrm{~PB} 1-\mathrm{KO}$ and $A P P / P S 1$ were significantly enriched for genes linked to negative regulation of amyloid $\beta$ formation, including Apoe, Clu, Ntrk2 (receptor for BDNF), Prnp, and 
Rtn3 (reticulon-3, which is involved in the regulation of $\beta$-secretase 1 (BACE1), a transmembrane proteases that catalyzes the first step in the formation of $A \beta$ peptide from amyloid precursor protein) (Shi et al., 2014)) (Fig. 3H). Plexin-B1 deficiency also caused upregulation of genes related to cell metabolism, e.g., Laspartate transmembrane transport (Slcla2, Slcla3), glutamate catabolism (Glud1, Glul), and cytochromec oxidase activity (Cox8a, Cox4l1, Ndufa4) (Fig. 3H). In addition, lipid pathway genes were also significantly upregulated in Plexin-B1-deficient sc-1 reactive astrocytes, e.g., arachidonate-CoA ligase activity (Acsl6, Acsl3), so were genes involved in astrocyte projection (Aqp4, Slcla2, Atplb2), consistent with the morphological and cell distancing phenotypes observed in APP/PS1 PB1-KO mice (Fig. 3H). Finally, Plexin-B1 KO also induced genes linked to calcium-dependent phospholipase A2 activity (Pla2g7, $\operatorname{Prdx\sigma }$ ) and negative regulation of long-term synaptic potentiation (Fam107a, Ptn) (Fig. 3H).

The downregulated genes in PB1-KO sc-1 reactive astrocytes in APP/PS1 background were significantly enriched for GO terms of cell junction disassembly $(C l q a / b / c, C x 3 c r l)$, regulation of glial cell migration (P2ry12), and cell-matrix interaction (Itgal), consistent with the phenotypes of altered astrocyte organization in AD-associated glial nets (Fig. 3H). Furthermore, GO analysis supported an attenuated inflammatory milieu as a result of $\mathrm{PB} 1-\mathrm{KO}$, as genes concerning complement activation $(\mathrm{Clqa} / \mathrm{b} / \mathrm{c})$ and regulation of TNF superfamily cytokine production ( $C x 3 \operatorname{cr} 1$, FoxP 1$)$ were suppressed. In addition, Cxcl12 (also known as SDF-1), a chemokine shown to induce proliferation of astrocytes (Bonavia et al., 2003), was among the top downregulated genes by PB1-KO (Fig. 3G). Collectively, our transcriptomic analyses provided further evidence that Plexin-B1 deletion altered functional states of reactive astrocytes consistent with improved amyloid $\beta$ plaque clearance and reduced neuroinflammation.

\section{Plexin-B1 deficiency also alters transcriptional states in disease-associated microglia}

We next studied the scRNA-seq data of microglia, which could be partitioned into 11 distinct subclusters

(Fig. 4A). As disease-associated microglia (DAM) have been identified as a distinct population associated 
with AD (Keren-Shaul et al., 2017), we therefore surveyed the expression of DAM signature genes ( $\mathrm{Lpl}$, Cst7, Axl, Itgax, Spp1, Cd9, Ccl6, and Csf1), which all showed high expression in sc-9 (Fig. 4B), whereas homeostatic microglial genes ( $C x 3 c r 1, P 2 r y 12$, Tmem 119) were all downregulated in sc-9 as compared to other microglial subclusters (Fig. 4C). Hence, sc-9 microglia is representative of the DAM population in AD-affected brains.

The proportion of the DAM-like sc-9 microglia was affected by Plexin-B1 KO in APP/PS1 background (Fig. 4D). First, $A P P / P S 1$ caused a substantial expansion of sc-9 from $1 \%$ in $\mathrm{WT}$ to $4 \%$ in $A P P / P S 1$. Second, PB1-KO resulted in a partial restoration, with sc-9 reduced to $2 \%$ in $A P P / P S 1 \mathrm{~PB} 1-\mathrm{KO}$. Third, in the absence of AD pathology, Plexin-B1 KO alone did not cause overt changes of the sc-9 representation. These findings support mitigated neuroinflammation as a result of Plexin-B1 ablation in AD brains.

Despite a reduction in the population share of sc-9 microglia, Plexin-B1 deletion altered the transcriptomic profile of sc-9 DAM microglia in a way that signifies augmented microglial function. We identified more than 1,000 DEGs in sc-9 microglia in $A P P / P S 1$ PB1-KO compared to $A P P / P S 1$, with the vast majority being upregulated (Fig. 4E). Gene enrichment analysis showed that Plexin-B1 deletion led to induction of genes linked to enhanced microglial cell activation (Cx3crl, Clqa, Grn, Ifngra, Aifl (encoding Iba1), and Tnf), cell junction disassembly $(C 1 q b, C 1 q a, C 1 q c, C x 3 c r 1)$, and host defense response (Ubb, B2m, Tlr2) (Fig. 4F). In addition, genes associated with class II MHC protein complex binding (Cd81, Ywhae, Hsp90ab1), chemoattractant and purinergic receptor activity (Cx3cr1, Ccrl2, Ccr5, P2ry12, Gpr34), and endolysosomal lumen (Ctss, Ctsb, Lgmn) were also significantly upregulated in sc-9 microglia in the absence of Plexin-B1 (Fig. 4F).

Plexin-B1 deletion leads to lower plaque numbers, a shift towards dense-core type, and less neurotoxicity 
So far, IHC and scRNA-seq data demonstrated that Plexin-B1 ablation not only affected glial net structures, but also transcriptional responses in both reactive astrocytes and DAM cells that affect amyloid metabolism and neuroinflammation. We next examined how these changes may impact amyloid $\beta$ clearance, plaque development, and the associated neurotoxicity.

Histological inspection of amyloid plaques showed a significant reduction of plaque numbers in 6-monthold APP/PS1 PB1-KO mice as compared to age-matched APP/PS1 mice (Fig. 5A). Notably, the average size of the plaques also appeared smaller, with close to 50\% reduction (Fig. 5A). Size spectrum analysis confirmed that PB1-KO resulted in a shift of amyloid plaques towards smaller sizes (Fig. 5C).

We further surveyed the composition of amyloid plaques by performing co-staining using anti-A $\beta$ antibody 6E10, which labels most amyloid plaques, and Thio-S dye, which stains cores of amyloid plaques (Condello et al., 2015) (Fig. 5B). The co-staining method allowed us to classify plaques according their amyloid compaction type (Fig. 5D). In the cortex of $A P P / P S 1$ mice at 6 months, we found that close to $50 \%$ of the plaques were of fibrillar type, 35\% mixed, and 15\% dense-core, whereas in APP/PS1 PB1-KO mice, the composition was reversed, with 15\% fibrillar, 35\% mixed, and 50\% dense-core type (Fig. 5D). As densecore plaque formation is an outcome of efficient microglial phagocytosis of plaque material (Huang et al., 2021), the increased microglial coverage of plaques and the augmented microglial function may account for the shift towards dense-core type in APP/PS1 PB1-KO genotype.

Amyloid plaques are typically surrounded by a halo of dystrophic neuronal membranes (Condello et al., 2015). To gauge neurotoxicity of amyloid plaques in different genotype conditions, we performed IF for LAMP1, an autophagy and endo-lysosomal marker that is highly enriched in dystrophic neurites (Condello et al., 2011; Gowrishankar et al., 2015). We found a 50\% reduction of the number of LAMP1-labeled areas in the cortex of $A P P / P S 1$ PB1-KO vs. APP/PS1 mice (Fig 6A, B; Fig. S8), which echoes the overall 
reduced plaque burden by PB1-KO. The average size of LAMP1-marked halos around plaques was also reduced in $A P P / P S 1$ PB1-KO mice, as was the total area marked by LAMP1, which reflects both the number and the size of LAMP1-marked halos (Fig. 6B). Hence, the decreased neuritic dystrophy that we observed in $A P P / P S 1 \mathrm{~PB} 1-\mathrm{KO}$ mice is consistent with the notion that dense-core plaques reflect a macrophage-mediated confinement mechanism that limits dissemination of neurotoxic soluble A $\beta$ oligomers (Huang et al., 2021).

We next assessed the expression of the lysosome marker CD68 (Chistiakov et al., 2017), which labels active phagocytic microglia. We detected comparable ratios of $\mathrm{CD}^{+} 8^{+}$areas relative to $\mathrm{A} \beta$-labeled plaques in $A P P / P S 1$ PB1-KO and APP/PS1 mice (Fig. 6C), indicating that the phagocytic activity of microglia was not expanded, but confined to a smaller footprint in PB1-KO.

\section{Plexin-B1 deletion results in attenuated gliosis in tauopathy model of AD}

To investigate Plexin-B1's function in astrocyte activation in tau-driven neurodegeneration, we expanded our studies to a mouse model of tauopathy by crossing the TAU-PS19 line (Yoshiyama et al., 2007) with the Plexin-B1 KO allele. IF examination of sections of hippocampus and entorhinal cortex of 3-month-old TAU PB1-KO mice showed that Plexin-B1 deletion resulted in attenuated astrocytosis in areas with high accumulation of phospho-Tau (Fig. S9A). We also observed attenuated microgliosis in these areas (Fig. S9B), signifying reduced inflammatory milieu in the neurodegenerative areas. Similarly, scRNA-seq of hippocampal areas of 5-month-old cohort confirmed a shrinkage of the reactive astrocyte population (Ast0 ) in the $T A U$ PB1-KO compared to $T A U$ mice (Fig. S10). Thus, the role of Plexin-B1 in mediating astrocytic reactivity is not limited to amyloid-driven AD, but also applies to Tau-driven neurodegeneration. 


\section{DISCUSSION}

Mobilization of astrocytes and microglia in peri-plaque reactive glial nets is critical to clear $A \beta$, mitigate neuroinflammation, and limit neurotoxicity. A prerequisite to detect and engulf $A \beta$ amyloid material is adequate coverage by glial processes, which involves morphological and transcriptomic transformation of plaque-associated astrocytes and microglia, leading to amyloid clearance and plaque compaction, hence reduced neurotoxicity (Huang et al., 2021).

Our study revealed that Plexin-B1 induction in peri-plaque glial nets is involved in activation of glial cells and in imposing wide glial cell spacing around amyloid plaques. Interestingly, we observed that the coverage of plaques by glial processes was in APP/PS1 control AD mice lower than in APP/PS1 PB1-KO mice. The low access of glial cells in control AD conditions to $\mathrm{A} \beta$ deposits may support a detrimental cycle of poor $\mathrm{A} \beta$ clearance, more diffuse fibrillar plaques, increased neurotoxicity, recruitment of more glial cells, heightened inflammation, and thus a large footprint of reactive glial nets (Fig. 6D).

Remarkably, removing Plexin-B1 relaxed cell distancing of peri-plaque nets, thereby reversing the cycle: better astroglial and microglial coverage of $A \beta$ plaques, better $A \beta$ clearance, a shift towards dense-core plaques, less neurotoxicity, reduced the need for more glial recruitment, and a smaller footprint of glial nets. Interestingly, the smaller glial nets with fewer reactive astrocytes observed in $A P P / P S 1 \mathrm{~PB} 1-\mathrm{KO}$ mice may give the impression of a compromised glial response to plaques. However, the reduced plaque burden with higher proportion of dense-core plaques and decreased neuritic dystrophy in $A P P / P S 1$ PB1-KO mice collectively signify an improved efficiency of glial cells to clear A $\beta$. Indeed, the comparative scRNA-seq study of $A P P / P S 1$ and APP/PS1 PB1-KO brains revealed that Plexin-B1 deletion led to induction of a wide range of genes linked to $A \beta$ clearance in both astrocytes and microglia. With individual glial cells functioning more effectively in amyloid metabolism, the need to recruit more reactive glial cells towards 
plaque areas is alleviated, hence a smaller footprint of glial nets, and reduced neurotoxicity and neuroinflammation.

Although Plexin-B1 is predominantly expressed in astrocytes, its deletion also elicited transcriptional changes in microglia and improved microglial amyloid coverage. Although we cannot exclude a possible effect from low expression of Plexin-B1 in microglia, a more likely explanation is a secondary effect from astrocyte-microglia interactions and altered inflammatory milieu in glial nets. This is supported by cytokine expression changes in astrocytes as a result of PB1-KO (e.g., downregulation of Ccl12). Moreover, in absence of Plexin-B1, relaxed cell distancing of reactive astrocytes may physically permit closer access of microglia to plaques, in turn eliciting morphological and transcriptomic transformation of microglial cells to a DAM state (Keren-Shaul et al., 2017). In this context, it is important to be aware of the reciprocal influence of microglia on astrocytes; for example, Clqa and Tnf, two well-known microglia-derived factors that trigger inflammatory phenotype of astrocytes (Liddelow et al., 2017), were among the upregulated genes in DAM cells as a result of Plexin-B1 deletion.

Plexin signaling is known for growth cone collapse during neurodevelopment; and recent studies from our laboratory and others have also unveiled a role of Plexin in cell collision guidance (Deb Roy et al., 2017; Zhou et al., 2020). For instance, we showed that Plexin-B2, a paralog of Plexin-B1, acts in microglia to steer them away from colliding astrocytes during wound repair after spinal cord injury via a process termed contact inhibition of locomotion (CIL). CIL has also reported during cellular contact between osteoclasts and osteoblasts, via Plexin-B1 (Deb Roy et al., 2017). The phenotypes of the current study are consistent with a potential role of Plexin-B1 in regulating CIL in peri-plaque glial nets, thus imposing cell distancing.

The identification of the molecular and cellular identity of the activating signals for Plexin-B1 in astrocytes will require further studies. The canonical ligands for Plexin-B1 are class 4 semaphorins, a family of six 
members that are expressed in diverse cell types (Gurrapu and Tamagnone, 2016; Zhou et al., 2008). Interestingly, a recent study in a multiple sclerosis mouse model showed that Sema4D expressed by microglia can activate Plexin-B1 and -B2 receptors on astrocytes (Clark et al., 2021). Hence, it is conceivable that in $\mathrm{AD}$, Sema4D expressed by microglia may activate Plexin-B1 on astrocytes to increase cell spacing. It is noteworthy that a semaphorin-independent mechanosensitive function of Plexin-D1, a paralog of Plexin-B1, has recently been reported for detection of shear stress by endothelial cells (Mehta et al., 2020). Thus, Plexin-B1 may possibly also function to sense mechanical forces, such as from distorted interstitial space near plaques, a model requiring further investigation.

Future therapies targeting Plexin-B1 may alleviate AD pathophysiology. Small molecule inhibitors of plexin signaling are currently unavailable, however, a function-blocking antibody against Sema4D is being tested in a clinical trial for Alzheimer's disease (Evans et al., 2020). Another viable approach to block Plexin-B1 is to employ cyclic peptides that act as allosteric inhibitors (Matsunaga et al., 2016).

In summary, our data demonstrate a role of reactive astrocytes, through guidance receptor Plexin-B1, in imposing cell distancing in peri-plaque glial nets, leading to restricted microglial coverage of A $\beta$ aggregates, reduced amyloid compaction, and widened neural tissue damage. Inhibition of Plexin-B1 function may represent an alternative therapeutic approach to reverse this process, thus improving disease outcome for AD patients. 


\section{Acknowledgements}

This research project was supported by the National Institute of Aging (NIH-NIA) grants U01AG046170 and RF1AG057440. We thank Michelle Ehrlich at Icahn School of Medicine at Mount Sinai for advice and sharing AD mice.

\section{Data availability}

The human postmortem sequencing data are available via the AD Knowledge Portal (https://adknowledgeportal.synapse.org). The AD Knowledge Portal is a platform for accessing data, analyses, and tools generated by the Accelerating Medicines Partnership (AMP-AD) Target Discovery Program and other National Institute on Aging (NIA)-supported programs to enable open-science practices and accelerate translational learning. The data, analyses, and tools are shared early in the research cycle without a publication embargo on a secondary use. Data is available for general research use according to the following requirements for data access and data attribution (https://adknowledgeportal.synapse.org/DataAccess/Instructions).

RNA-seq data sets generated by this study have been deposited at the AMP-AD knowledge portal under Synapse ID syn26076878 (https://www.synapse.org/\#!Synapse:syn26076878). 


\section{METHODS}

\section{Mice}

All animal procedures were conducted in accordance with protocols approved by the Institutional Animal Care and Use Committee (IACUC) of Icahn School of Medicine at Mount Sinai. Mice were bred on C57BL/6J genetic background.

Mouse mutant lines are as follows:

APP/PS1 transgenic line Tg(APPswe,PSEN1dE9)85Dbo (Jankowsky et al., 2004), JAX strain MMRRC_034829-JAX;

TAU (TAU-PS19) transgenic line Tg(Prnp-MAPT*P301S)PS19Vle (Yoshiyama et al., 2007), JAX strain \#008169;

Plexin-B1 KO line Plxnb1 ${ }^{\text {tm1Matl }}$ (Daviaud et al., 2016), MGI allele MGI:3765917; this KO line carries a lacZ-IRES-PLAP reporter inserted into the Plxnb1 locus (Friedel et al., 2005).

The $A P P / P S 1$ transgene insertion is located about $4 \mathrm{Mb}$ away from the Plxnb1 gene locus on chromosome 9, which creates a partial linkage of Plxnbl with the APP/PS1 transgene, and breeding schemes were adjusted in some cases by using cis-heterozygous $A P P / P S 1 /+P l x n b 1^{+/-}$breeders.

\section{Histology}

For analysis of post-mortem brain tissue from de-identified AD patients, microtome sections of FFPE (formalin-fixed paraffin-embedded) tissue blocks with associated information about patient age, clinical symptoms, and ABC neuropathology score (Hyman et al., 2012; Montine et al., 2012) were provided by the Mount Sinai Neuropathology Brain Bank. Immunohistochemistry (IHC) was performed with a Ventana staining system (Roche). Primary antibodies used for IHC are as follows:

anti-Plexin-B1 (host: rabbit, Sigma HPA040586, used at 1:50 dilution), anti-GFAP (host: mouse, Takara Y40420 (STEM123), 1:1000), 
anti-A $\beta$ amyloid (host: mouse, clone 4G8, BioLegend 800701, 1:100).

For analysis of mouse brain tissues, cryopreserved brains were sectioned on a cryostat at $20 \mu \mathrm{m}$ thickness and floating sections were collected in PBS and stored at $4^{\circ} \mathrm{C}$. For immunofluorescence (IF) staining, sections were incubated with blocking buffer (5\% normal donkey serum (Jackson ImmunoResearch) and $0.3 \%$ Triton $\mathrm{X}-100$ in $\mathrm{PBS}$ ) for one hour at room temperature prior to overnight incubation at $4^{\circ} \mathrm{C}$ with primary antibodies in antibody-dilution buffer (1\% BSA (Fisher Bioreagents) and 0.3\% Triton X-100 in PBS).

The following antibodies were used for IF:

anti- $\beta$-amyloid (host: mouse, clone 6E10, BioLegend 803015, 1:800),

anti-IBA1 (host: rabbit, Wako Laboratory Chemicals 019-19741, 1:500),

anti-IBA1 goat (Novus Biologics NB100-1028) (1:250),

anti-GFAP (host: rabbit, Dako Z0334, 1:800),

anti-LAMP1 (host: rat, clone 1D4B, DSHB, 1:200),

anti-CD68 (host: rat, clone FA-11, Bio-Rad MCA1957GA, 1: 500),

anti-phospho-TAU (S202, T205) (host: mouse, clone AT8, Thermo Fisher MN1020, 1: 50).

Antigen retrieval was performed prior to immunostaining for amyloid and tau aggregates. Floating sections were treated with 50\% formic acid (Sigma F0507, diluted with distilled water) for 20 minutes and then washed three times with PBS (5 minutes per wash). Subsequently, phospho-Tau or amyloid-beta protein (A $\beta)$ was detected by IF.

Thioflavin S (Sigma T1892) staining of amyloid plaques was performed by staining floating brain sections after IF with $0.005 \%$ Thioflavin S solution (in tris-buffered saline, TBS) for 8 minutes. Differentiation of the dye was carried out by two rounds of incubation in $50 \%$ ethanol for one minute each. Sections were 
washed for 5 minutes in TBS and then mounted with Fluoromount-G mounting medium.

\section{X-Gal and Congo-Red staining}

$\beta$-galactosidase reporter expression was identified histochemically by X-Gal assay. X-gal staining solution was prepared by adding X-Gal (dissolved in dimethylformamide) at $1 \mathrm{mg} / \mathrm{ml}$ to staining buffer $(0.02 \%$ Igepal, $0.01 \%$ sodium deoxycholate, $5 \mathrm{mM}$ potassium ferricyanide, $5 \mathrm{mM}$ potassium ferrocyanide, and 2 $\mathrm{mM} \mathrm{MgCl2}$ diluted in 0.1 M PBS (pH 7.3)). 6-month-old APP mice were perfused with 4\% PFA/PBS, brains were post-fixed for 6 hours in and embedded in 4\% agarose/PBS for vibratome sectioning and stored at $4{ }^{\circ} \mathrm{C} .100 \mu \mathrm{m}$ thick brain sections were collected and were incubated overnight in X-gal staining solution at $37^{\circ} \mathrm{C}$. Slides were then dipped sequentially in $70 \%$ ethanol for 5 minutes, followed by $100 \%$ ethanol for 10 minutes and lastly $70 \%$ ethanol for 1 minute before hydrating them in water. Slides were then incubated in a $1 \%$ aqueous Congo Red solution (Sigma C6277) for 30-60 minutes at RT followed by rapid differentiation (5-10 dips) in alkaline alcohol solution $(1 \% \mathrm{NaOH}, 50 \%$ ethanol), and rinsed in running water for 10 minutes.

\section{Astrocyte cell culture assays}

Primary astrocyte cultures were generated from post-natal day 2 or 3 pups. Briefly, forebrains were dissected and triturated. Cells were plated in DMEM media with 10\% FBS and 1:100 Pen/Strep (Gibco) and then serially passaged for at least three times to enrich for astrocytes. Astrocyte cultures were further enriched using an anti-ACSA-2 MicroBead Kit (Milteny Biotec \#130-097-679). Briefly, primary astrocytes were prepared in single cell suspension, incubated with anti-ACSA-2 microbeads and separated with MS magnetic columns (Milteny Biotec \#130-042-201).

For treatment of cells with oligomerized A $\beta$ amyloid (oA $\beta$ ), $\beta$ amyloid (1-42) (Bachem \#4061966) was dissolved in hexafluoroisopropanol (MP Biomedicals \#0215124505) to obtain a $1 \mathrm{mM}$ solution. Solvent was evaporated from the solution by SpeedVac centrifugation. Dried A $\beta$ amyloid was re-suspended in 
DMSO to obtain a $5 \mathrm{mM}$ solution, sonicated in water bath for 10 minutes, diluted in PBS to a concentration of $50 \mu \mathrm{M}$, and stored at $4^{\circ} \mathrm{C}$ for 12 hours before adding to the cells.

For ApoE ELISA, astrocytes were seeded in 24 well plates at a density of 30,000 cells per well. After 24 hours, cells were cultured with serum free DMEM medium with vehicle (0), 2, 5, and $10 \mu \mathrm{M}$ oA $\beta$. After 24 hours, supernatant was collected and secretion of ApoE protein into the cell culture supernatant was measured with an ApoE ELISA kit (Mybiosource \#MBS705227).

\section{Bulk RNA sequencing}

RNA-seqencing was performed using total RNAs isolated from freshly dissected brain tissues using the RNeasy Mini Plus kit (Qiagen). TruSeq stranded cDNA libraries and 100-nucleotide paired reads with the Illumina Novaseq6000 S4 system were generated at Psomagen, Inc.. Sequencing reads were aligned to a customized mouse reference genome mm10 using STAR aligner v2.5.3a (Dobin et al., 2013) guided by a customized mouse GENCODE gene model release v15. Here, the customized mouse reference genome sequence file was prepared based on the mm10 sequence by inserting 3 pseudo chromosomes, containing human APP, PSEN1, or MAPT gene sequences, respectively. Meanwhile, the GENCODE gene annotation GTF file was customized by adding annotations corresponding to human APP, PSEN1, and MAPT transgenes so that the expression of human APP/PSEN1/MAPT genes in the transgenic mice could be quantified. Mapped reads were summarized to gene levels using the featureCounts program v1.6.3 (Liao et al., 2014). Raw count data were normalized by the voom function in the R limma package (Ritchie et al., 2015) and then differential expression was called between sample conditions by the moderated t-test implemented in limma. Differentially expressed genes (DEGs) were defined to have at least 1.2-fold change in expression and Benjamini-Hochberg (BH) (Benjamini and Hochberg, 1995) adjusted $P \leq 0.05$.

\section{Single-cell sequencing}

scRNA-seq was performed using the 10X Genomics system at the Mount Sinai Genomics core facility. To 
prepare single cell suspensions for library preparation, fresh brain tissues were dissociated using a Papain neural tissue dissociation kit (Miltenyi 130-092-628).

The scRNA-seq data were analyzed using the Seurat package (Stuart et al., 2019). Starting from Cell Ranger derived unique molecular identifier (UMI) count matrices, quality control (QC) was first performed by removing cells with either too few genes $(\leq 200)$, too many genes $(\geq 5000)$, an excessive number of UMI counts $(\geq 50,000)$, or more than $10 \%$ mitochondrial reads, retaining 27,286 cells after filtering. Meanwhile, insufficiently detected genes were removed by keeping 18,762 genes expressed in more than two cells. After QC, we utilized Seurat's canonical correlation analysis (CCA) pipeline to integrate data across sample conditions. Next, dimensional reduction was performed using principal component analysis (PCA). The significant principal components explaining more than $90 \%$ of variance were selected for cell clustering using Seurat's graph-based clustering approach. We projected the normalized dataset onto a 2D space determined by Uniform Manifold Approximation and Projection for Dimension Reduction (UMAP) (McInnes et al., 2018). For each cluster, we calculated cluster gene signatures by comparing cells in one cluster against cells of remaining clusters using Wilcox rank sum test. Next, we interrogated the expression patterns of known marker genes to associate clusters with major cell-types: neurons (Gad1, Slc17a7), astrocytes (Aqp4), oligodendrocytes (Mog), microglia (Tmem119, Trem2, and Tyrobp), oligodendrocyte progenitor cells (Vcan), endothelial cells (Flt1), and pericytes (Ambp).

To identify subclusters of astrocytes or microglia, cells from the astrocyte or microglia clusters defined above were extracted as subset. Then dimensional reduction was again performed using PCA, with the top principal components selected for cell clustering by Seurat's graph-based clustering approach.

\section{Data base analyses}

Analysis of the expression of Plexin-B1 in cell types of the CNS was performed at the Brain RNA-seq platform (www.brainrnaseq.org) (Zhang et al., 2014; Zhang et al., 2016).

The enrichment of Plexin-B1 network genes was performed against CNS cell type marker genes (defined 
in ref. (McKenzie et al., 2018)) and the MSigDB signature database (Liberzon et al., 2015; Subramanian et al., 2005) with Fisher exact test.

Gene ontology enrichment analysis for sets of differentially expressed genes was performed with the Enrichr platform (Xie et al., 2021) (maayanlab.cloud/Enrichr/).

\section{Statistical analysis}

Statistical analyses were performed with GraphPad Prism 9 software. Bar graph data are presented as mean \pm SEM. 


\section{References}

Bonavia, R., Bajetto, A., Barbero, S., Pirani, P., Florio, T., and Schettini, G. (2003). Chemokines and their receptors in the CNS: expression of CXCL12/SDF-1 and CXCR4 and their role in astrocyte proliferation. Toxicol Lett 139, 181-189.

Bos, J.L. (2018). From Ras to Rap and Back, a Journey of 35 Years. Cold Spring Harb Perspect Med 8.

Bouvier, D.S., Jones, E.V., Quesseveur, G., Davoli, M.A., A Ferreira, T., Quirion, R., Mechawar, N., and Murai, K.K. (2016). High Resolution Dissection of Reactive Glial Nets in Alzheimer's Disease. Sci Rep 6, 24544.

Ceyzériat, K., Ben Haim, L., Denizot, A., Pommier, D., Matos, M., Guillemaud, O., Palomares, M.A., Abjean, L., Petit, F., Gipchtein, P., et al. (2018). Modulation of astrocyte reactivity improves functional deficits in mouse models of Alzheimer's disease. Acta Neuropathol Commun 6, 104.

Chistiakov, D.A., Killingsworth, M.C., Myasoedova, V.A., Orekhov, A.N., and Bobryshev, Y.V. (2017). CD68/macrosialin: not just a histochemical marker. Lab Invest 97, 4-13.

Clark, I.C., Gutiérrez-Vázquez, C., Wheeler, M.A., Li, Z., Rothhammer, V., Linnerbauer, M., Sanmarco, L.M., Guo, L., Blain, M., Zandee, S.E.J., et al. (2021). Barcoded viral tracing of single-cell interactions in central nervous system inflammation. Science 372.

Condello, C., Schain, A., and Grutzendler, J. (2011). Multicolor time-stamp reveals the dynamics and toxicity of amyloid deposition. Sci Rep 1, 19.

Condello, C., Yuan, P., Schain, A., and Grutzendler, J. (2015). Microglia constitute a barrier that prevents neurotoxic protofibrillar A $\beta 42$ hotspots around plaques. Nat Commun 6, 6176.

Daviaud, N., Chen, K., Huang, Y., Friedel, R.H., and Zou, H. (2016). Impaired cortical neurogenesis in plexin-B1 and -B2 double deletion mutant. Dev Neurobiol 76, 882-899.

Deb Roy, A., Yin, T., Choudhary, S., Rodionov, V., Pilbeam, C.C., and Wu, Y.I. (2017). Optogenetic activation of Plexin-B1 reveals contact repulsion between osteoclasts and osteoblasts. Nat Commun $8,15831$.

Deng, S., Hirschberg, A., Worzfeld, T., Penachioni, J.Y., Korostylev, A., Swiercz, J.M., Vodrazka, P., Mauti, O., Stoeckli, E.T., Tamagnone, L., et al. (2007). Plexin-B2, but not Plexin-B1, critically modulates neuronal migration and patterning of the developing nervous system in vivo. J Neurosci 27, 6333-6347.

Dobin, A., Davis, C.A., Schlesinger, F., Drenkow, J., Zaleski, C., Jha, S., Batut, P., Chaisson, M., and Gingeras, T.R. (2013). STAR: ultrafast universal RNA-seq aligner. Bioinformatics 29, 15-21.

Evans, E.E., Fisher Jr., T.L., Leonard, J.E., Reader, A.M., Mishra, V., Mallow, C.L., Balch, L., Howell, A., Smith, E.S., Feigin, A., et al. (2020). Evidence that semaphorin 4D is upregulated in neurons in Huntington's and Alzheimer's diseases: Effects of a SEMA4D blocking antibody on FDG-PET in a clinical trial, and treatment rationale for its use in AD. Alzheimer's \& Dementia 16, e043971.

Fazzari, P., Penachioni, J., Gianola, S., Rossi, F., Eickholt, B.J., Maina, F., Alexopoulou, L., Sottile, A., Comoglio, P.M., Flavell, R.A., et al. (2007). Plexin-B1 plays a redundant role during mouse development and in tumour angiogenesis. BMC Dev Biol 7, 55.

Friedel, R.H., Plump, A., Lu, X., Spilker, K., Jolicoeur, C., Wong, K., Venkatesh, T.R., Yaron, A., Hynes, M., Chen, B., et al. (2005). Gene targeting using a promoterless gene trap vector ("targeted trapping") is an efficient method to mutate a large fraction of genes. Proc Natl Acad Sci U S A 102, 13188-13193. 
Gandy, S., and DeKosky, S.T. (2013). Toward the treatment and prevention of Alzheimer's disease: rational strategies and recent progress. Annu Rev Med 64, 367-383.

Gandy, S., and Ehrlich, M.E. (2021). Alzheimer mutant speeds APP transport. J Exp Med 218.

Gowrishankar, S., Yuan, P., Wu, Y., Schrag, M., Paradise, S., Grutzendler, J., De Camilli, P., and Ferguson, S.M. (2015). Massive accumulation of luminal protease-deficient axonal lysosomes at Alzheimer's disease amyloid plaques. Proc Natl Acad Sci U S A 112, E3699-3708.

Gurrapu, S., and Tamagnone, L. (2016). Transmembrane semaphorins: Multimodal signaling cues in development and cancer. Cell Adh Migr 10, 675-691.

Hota, P.K., and Buck, M. (2012). Plexin structures are coming: opportunities for multilevel investigations of semaphorin guidance receptors, their cell signaling mechanisms, and functions. Cell Mol Life Sci 69, 3765-3805.

Huang, Y., Happonen, K.E., Burrola, P.G., O'Connor, C., Hah, N., Huang, L., Nimmerjahn, A., and Lemke, G. (2021). Microglia use TAM receptors to detect and engulf amyloid $\beta$ plaques. Nat Immunol 22, 586-594.

Hyman, B.T., Phelps, C.H., Beach, T.G., Bigio, E.H., Cairns, N.J., Carrillo, M.C., Dickson, D.W., Duyckaerts, C., Frosch, M.P., Masliah, E., et al. (2012). National Institute on Aging-Alzheimer's Association guidelines for the neuropathologic assessment of Alzheimer's disease. Alzheimers Dement 8, 1-13.

Jankowsky, J.L., Fadale, D.J., Anderson, J., Xu, G.M., Gonzales, V., Jenkins, N.A., Copeland, N.G., Lee, M.K., Younkin, L.H., Wagner, S.L., et al. (2004). Mutant presenilins specifically elevate the levels of the 42 residue betaamyloid peptide in vivo: evidence for augmentation of a 42-specific gamma secretase. Hum Mol Genet 13, 159170.

Jongbloets, B.C., and Pasterkamp, R.J. (2014). Semaphorin signalling during development. Development 141, 3292-3297.

Junqueira Alves, C., Silva Ladeira, J., Hannah, T., Pedroso Dias, R.J., Zabala Capriles, P.V., Yotoko, K., Zou, H., and Friedel, R.H. (2021). Evolution and Diversity of Semaphorins and Plexins in Choanoflagellates. Genome Biol Evol 13.

Keren-Shaul, H., Spinrad, A., Weiner, A., Matcovitch-Natan, O., Dvir-Szternfeld, R., Ulland, T.K., David, E., Baruch, K., Lara-Astaiso, D., Toth, B., et al. (2017). A Unique Microglia Type Associated with Restricting Development of Alzheimer's Disease. Cell 169, 1276-1290.e1217.

Koropouli, E., and Kolodkin, A.L. (2014). Semaphorins and the dynamic regulation of synapse assembly, refinement, and function. Curr Opin Neurobiol 27, 1-7.

Leng, F., and Edison, P. (2021). Neuroinflammation and microglial activation in Alzheimer disease: where do we go from here? Nat Rev Neurol 17, 157-172.

Liao, Y., Smyth, G.K., and Shi, W. (2014). featureCounts: an efficient general purpose program for assigning sequence reads to genomic features. Bioinformatics 30, 923-930.

Liberzon, A., Birger, C., Thorvaldsdóttir, H., Ghandi, M., Mesirov, J.P., and Tamayo, P. (2015). The Molecular Signatures Database (MSigDB) hallmark gene set collection. Cell systems 1, 417-425.

Liddelow, S.A., Guttenplan, K.A., Clarke, L.E., Bennett, F.C., Bohlen, C.J., Schirmer, L., Bennett, M.L., Münch, A.E., Chung, W.S., Peterson, T.C., et al. (2017). Neurotoxic reactive astrocytes are induced by activated microglia. Nature 541, 481-487. 
Long, J.M., and Holtzman, D.M. (2019). Alzheimer Disease: An Update on Pathobiology and Treatment Strategies. Cell 179, 312-339.

Matejuk, A., and Ransohoff, R.M. (2020). Crosstalk Between Astrocytes and Microglia: An Overview. Front Immunol 11, 1416.

Mathys, H., Davila-Velderrain, J., Peng, Z., Gao, F., Mohammadi, S., Young, J.Z., Menon, M., He, L., Abdurrob, F., Jiang, X., et al. (2019). Single-cell transcriptomic analysis of Alzheimer's disease. Nature 570, 332-337.

Matsunaga, Y., Bashiruddin, N.K., Kitago, Y., Takagi, J., and Suga, H. (2016). Allosteric Inhibition of a Semaphorin 4D Receptor Plexin B1 by a High-Affinity Macrocyclic Peptide. Cell Chem Biol 23, 1341-1350.

McInnes, L., Healy, J., and Melville, J. (2018). UMAP: Uniform Manifold Approximation and Projection for Dimension Reduction. arXiv 1802.03426.

McKenzie, A.T., Wang, M., Hauberg, M.E., Fullard, J.F., Kozlenkov, A., Keenan, A., Hurd, Y.L., Dracheva, S., Casaccia, P., Roussos, P., et al. (2018). Brain Cell Type Specific Gene Expression and Co-expression Network Architectures. Scientific reports 8,8868 .

Mehta, V., Pang, K.L., Rozbesky, D., Nather, K., Keen, A., Lachowski, D., Kong, Y., Karia, D., Ameismeier, M., Huang, J., et al. (2020). The guidance receptor plexin D1 is a mechanosensor in endothelial cells. Nature 578, 290295.

Montine, T.J., Phelps, C.H., Beach, T.G., Bigio, E.H., Cairns, N.J., Dickson, D.W., Duyckaerts, C., Frosch, M.P., Masliah, E., Mirra, S.S., et al. (2012). National Institute on Aging-Alzheimer's Association guidelines for the neuropathologic assessment of Alzheimer's disease: a practical approach. Acta Neuropathol 123, 1-11.

Mostafavi, S., Gaiteri, C., Sullivan, S.E., White, C.C., Tasaki, S., Xu, J., Taga, M., Klein, H.U., Patrick, E., Komashko, V., et al. (2018). A molecular network of the aging human brain provides insights into the pathology and cognitive decline of Alzheimer's disease. Nat Neurosci 21,811-819.

Neff, R.A., Wang, M., Vatansever, S., Guo, L., Ming, C., Wang, Q., Wang, E., Horgusluoglu-Moloch, E., Song, W.M., Li, A., et al. (2021). Molecular subtyping of Alzheimer's disease using RNA sequencing data reveals novel mechanisms and targets. Sci Adv 7.

Negishi-Koga, T., Shinohara, M., Komatsu, N., Bito, H., Kodama, T., Friedel, R.H., and Takayanagi, H. (2011). Suppression of bone formation by osteoclastic expression of semaphorin 4D. Nat Med 17, 1473-1480.

Okuno, T., Nakatsuji, Y., Moriya, M., Takamatsu, H., Nojima, S., Takegahara, N., Toyofuku, T., Nakagawa, Y., Kang, S., Friedel, R.H., et al. (2010). Roles of Sema4D-Plexin-B1 Interactions in the Central Nervous System for Pathogenesis of Experimental Autoimmune Encephalomyelitis. J Immunol 184, 1499-1506.

Ritchie, M.E., Phipson, B., Wu, D., Hu, Y., Law, C.W., Shi, W., and Smyth, G.K. (2015). limma powers differential expression analyses for RNA-sequencing and microarray studies. Nucleic Acids Res 43, e47.

Shi, Q., Ge, Y., Sharoar, M.G., He, W., Xiang, R., Zhang, Z., Hu, X., and Yan, R. (2014). Impact of RTN3 deficiency on expression of BACE1 and amyloid deposition. J Neurosci 34, 13954-13962.

Smit, T., Deshayes, N.A.C., Borchelt, D.R., Kamphuis, W., Middeldorp, J., and Hol, E.M. (2021). Reactive astrocytes as treatment targets in Alzheimer's disease-Systematic review of studies using the APPswePS1dE9 mouse model. Glia 69, 1852-1881.

Stuart, T., Butler, A., Hoffman, P., Hafemeister, C., Papalexi, E., Mauck, W.M., Hao, Y., Stoeckius, M., Smibert, P., and Satija, R. (2019). Comprehensive Integration of Single-Cell Data. Cell 177, 1888-1902.e1821. 
Subramanian, A., Tamayo, P., Mootha, V.K., Mukherjee, S., Ebert, B.L., Gillette, M.A., Paulovich, A., Pomeroy, S.L., Golub, T.R., Lander, E.S., et al. (2005). Gene set enrichment analysis: a knowledge-based approach for interpreting genome-wide expression profiles. Proc Natl Acad Sci U S A 102, 15545-15550.

Takamatsu, H., Takegahara, N., Nakagawa, Y., Tomura, M., Taniguchi, M., Friedel, R.H., Rayburn, H., TessierLavigne, M., Yoshida, Y., Okuno, T., et al. (2010). Semaphorins guide the entry of dendritic cells into the lymphatics by activating myosin II. Nat Immunol 11, 594-600.

Tran, T.S., Kolodkin, A.L., and Bharadwaj, R. (2007). Semaphorin regulation of cellular morphology. Annu Rev Cell Dev Biol 23, 263-292.

Wang, M., Beckmann, N.D., Roussos, P., Wang, E., Zhou, X., Wang, Q., Ming, C., Neff, R., Ma, W., Fullard, J.F., et al. (2018). The Mount Sinai cohort of large-scale genomic, transcriptomic and proteomic data in Alzheimer's disease. Sci Data 5, 180185.

Wang, M., Li, A., Sekiya, M., Beckmann, N.D., Quan, X., Schrode, N., Fernando, M.B., Yu, A., Zhu, L., Cao, J., et al. (2020). Transformative Network Modeling of Multi-Omics Data Reveals Detailed Circuits, Key Regulators, and Potential Therapeutics for Alzheimer's Disease. Neuron S0896-6273, 8.

Wang, M., Li, A., Sekiya, M., Beckmann, N.D., Quan, X., Schrode, N., Fernando, M.B., Yu, A., Zhu, L., Cao, J., et al. (2021). Transformative Network Modeling of Multi-omics Data Reveals Detailed Circuits, Key Regulators, and Potential Therapeutics for Alzheimer's Disease. Neuron 109, 257-272.e214.

Xie, Z., Bailey, A., Kuleshov, M.V., Clarke, D.J.B., Evangelista, J.E., Jenkins, S.L., Lachmann, A., Wojciechowicz, M.L., Kropiwnicki, E., Jagodnik, K.M., et al. (2021). Gene Set Knowledge Discovery with Enrichr. Curr Protoc 1, e90.

Yoshiyama, Y., Higuchi, M., Zhang, B., Huang, S.M., Iwata, N., Saido, T.C., Maeda, J., Suhara, T., Trojanowski, J.Q., and Lee, V.M. (2007). Synapse loss and microglial activation precede tangles in a P301S tauopathy mouse model. Neuron 53, 337-351.

Yu, L., Petyuk, V.A., Gaiteri, C., Mostafavi, S., Young-Pearse, T., Shah, R.C., Buchman, A.S., Schneider, J.A., Piehowski, P.D., Sontag, R.L., et al. (2018). Targeted brain proteomics uncover multiple pathways to Alzheimer's dementia. Ann Neurol 84, 78-88.

Yuan, P., Condello, C., Keene, C.D., Wang, Y., Bird, T.D., Paul, S.M., Luo, W., Colonna, M., Baddeley, D., and Grutzendler, J. (2016). TREM2 Haplodeficiency in Mice and Humans Impairs the Microglia Barrier Function Leading to Decreased Amyloid Compaction and Severe Axonal Dystrophy. Neuron 92, 252-264.

Zhang, B., Gaiteri, C., Bodea, L.G., Wang, Z., McElwee, J., Podtelezhnikov, A.A., Zhang, C., Xie, T., Tran, L., Dobrin, R., et al. (2013). Integrated systems approach identifies genetic nodes and networks in late-onset Alzheimer's disease. Cell 153, 707-720.

Zhang, Y., Chen, K., Sloan, S.A., Bennett, M.L., Scholze, A.R., O'Keeffe, S., Phatnani, H.P., Guarnieri, P., Caneda, C., Ruderisch, N., et al. (2014). An RNA-sequencing transcriptome and splicing database of glia, neurons, and vascular cells of the cerebral cortex. J Neurosci 34, 11929-11947.

Zhang, Y., Sloan, S.A., Clarke, L.E., Caneda, C., Plaza, C.A., Blumenthal, P.D., Vogel, H., Steinberg, G.K., Edwards, M.S., Li, G., et al. (2016). Purification and Characterization of Progenitor and Mature Human Astrocytes Reveals Transcriptional and Functional Differences with Mouse. Neuron 89, 37-53.

Zhou, X., Wahane, S., Friedl, M.S., Kluge, M., Friedel, C.C., Avrampou, K., Zachariou, V., Guo, L., Zhang, B., $\mathrm{He}$, X., et al. (2020). Microglia and macrophages promote corralling, wound compaction and recovery after spinal cord injury via Plexin-B2. Nat Neurosci 23, 337-350. 
Zhou, Y., Gunput, R.A., and Pasterkamp, R.J. (2008). Semaphorin signaling: progress made and promises ahead. Trends Biochem Sci 33, 161-170. 

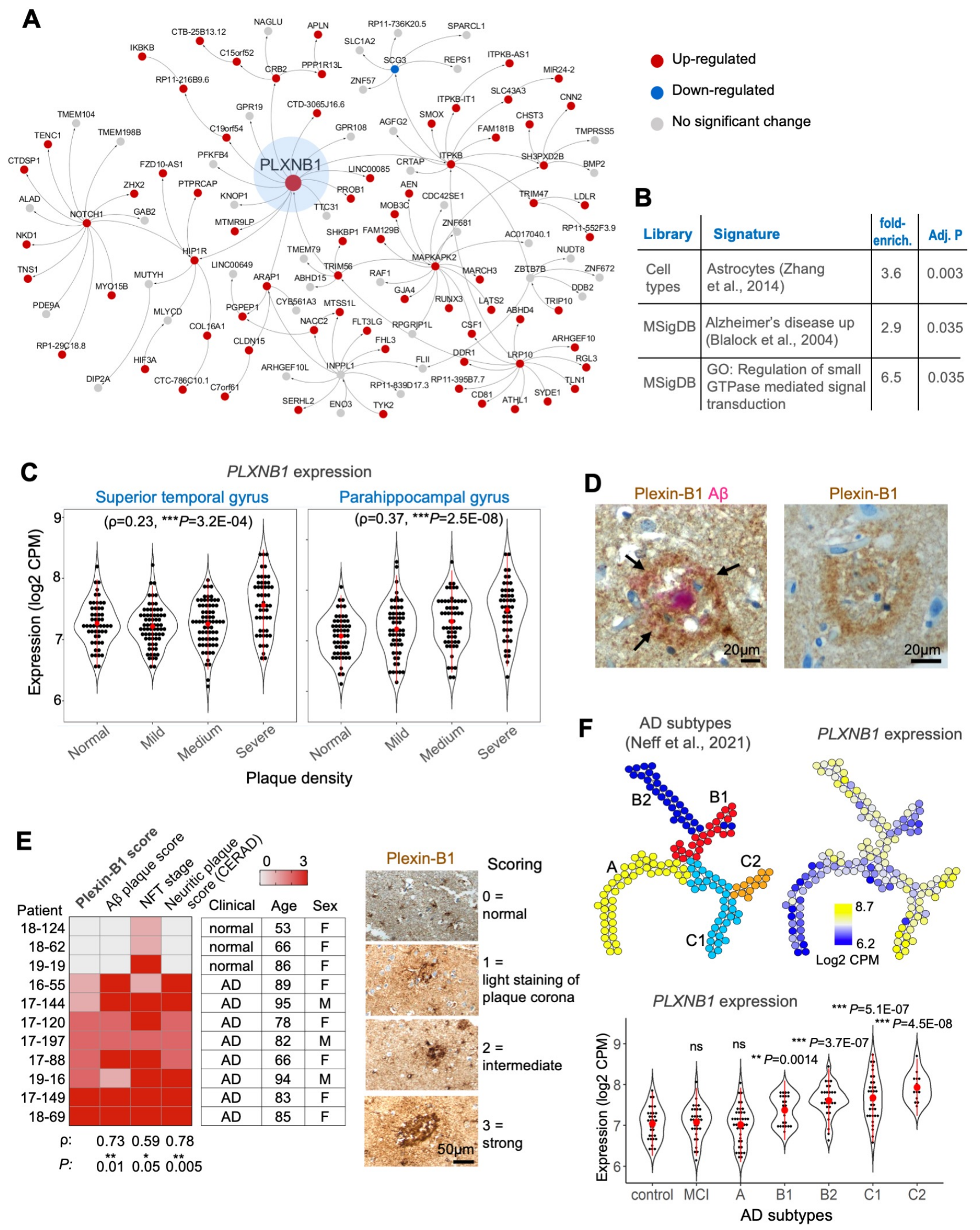

Figure 1. Plexin-B1 upregulation is plaque-associated and correlates with disease severity in human AD patients. 
A) Multiscale gene network analysis of large -omics data of AD patients identified PLXNB1 as a hub in a coregulated gene subnetwork underlying late-onset AD (Mount Sinai Brain Bank cohort).

B) Top significant hits of enrichment analysis of $P L X N B 1$-associated genes in the gene module identified in lateonset $\mathrm{AD}$ patients for Cell type and MSigDB signatures. The identifier of the gene ontology (GO) term is GO:0051056.

C) Spearman's Rho $(\rho)$ rank correlation analyses show that PLXNB1 mRNA levels in the two indicated brain regions are positively correlated with plaque density in $\mathrm{AD}$ patients.

D) IHC images from post-mortem brain sections of 66-year-old AD patient with neuropathology score of A3B3C2 reveal elevated Plexin-B1 protein expression in a corona-like pattern (arrows) surrounding amyloid plaque (A $\beta$ antibody 4G8). The image to the right is example of IHC with only anti-Plexin-B1 antibody. Hematoxylin was used as nuclear counterstain.

E) Heatmap of comparative correlation of Plexin-B1 expression scores and neuropathological scores for 11 AD patients (Mount Sinai Brain Bank). Pearson correlation coefficient $(\rho)$ and $P$ values are listed at the bottom. Examples of the Plexin-B1 scoring scheme are illustrated on the right.

F) $P L X N B 1$ is most upregulated in $\mathrm{AD}$ subtypes $\mathrm{C} 1 / \mathrm{C} 2$. Top, each circle represents one patient from the Mount Sinai Brain Bank cohort ( $\mathrm{n}=151$ participants). Bottom, graphs of $P L X N B 1$ expression in parahippocampal gyrus in each AD subtypes. One-tailed $t$-test. 
A
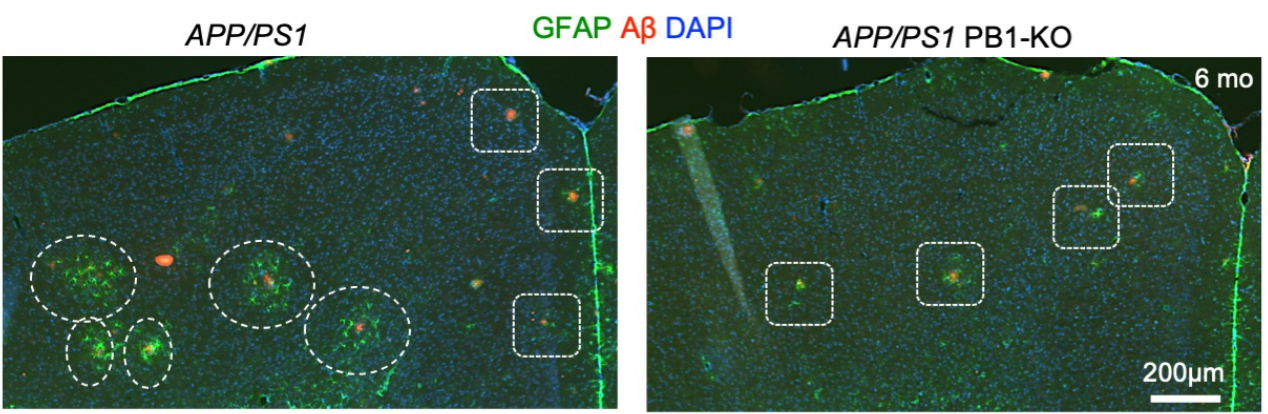

B
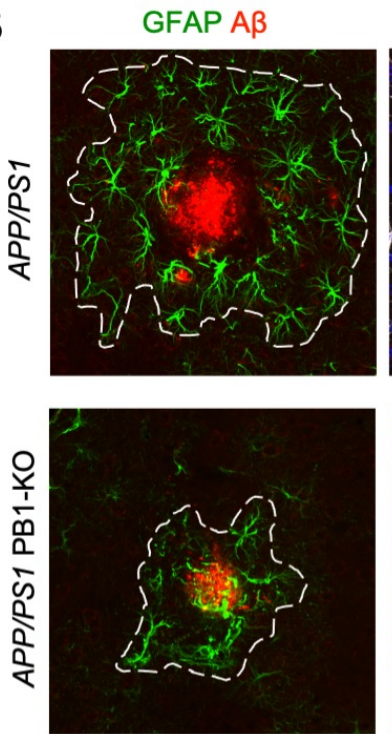

A $\beta$ IBA1 DAPI
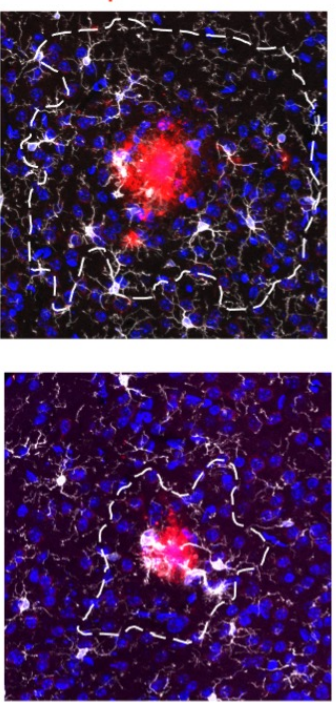
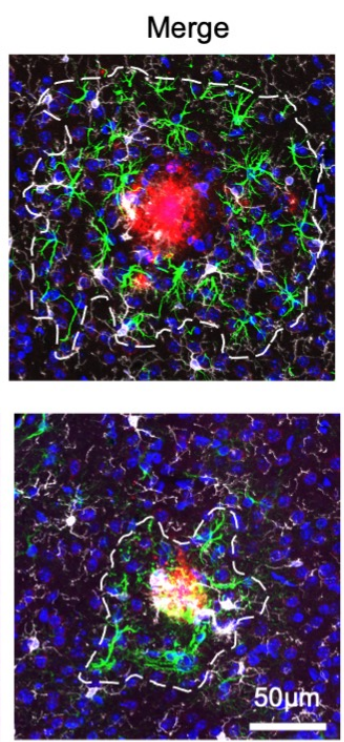

Glial net territory

(defined by perimeter of $\mathrm{GFAP}^{+}$cells)

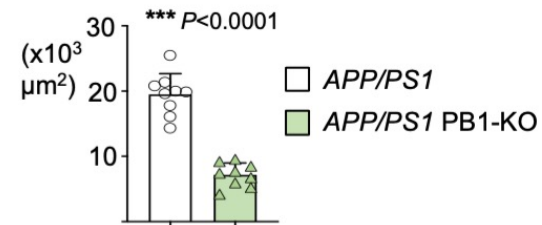

Distance to plaque margin by $\mathrm{GFAP}^{+}$cells

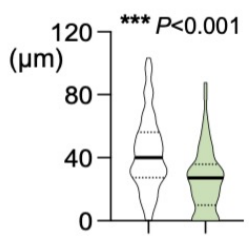

C

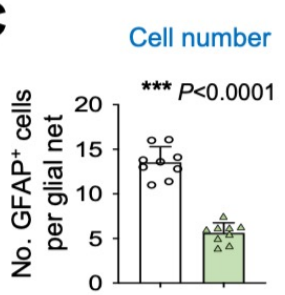

D

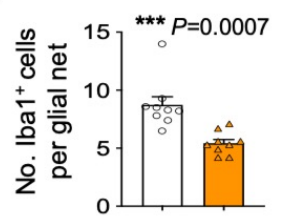

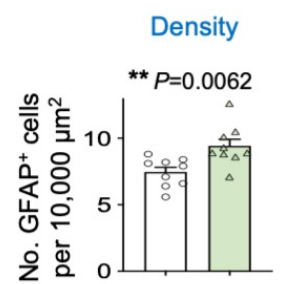

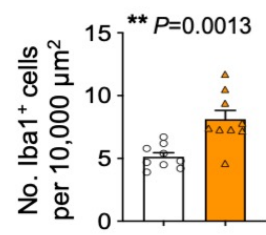

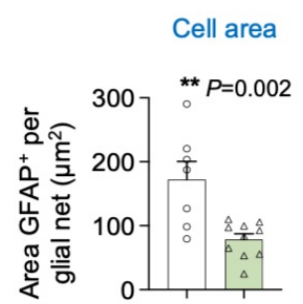

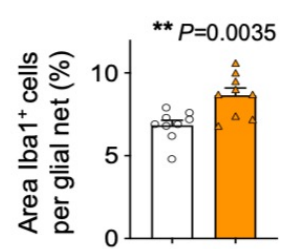

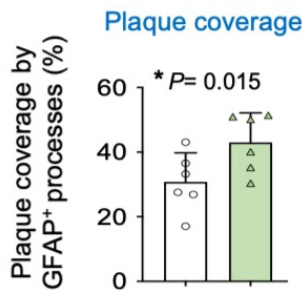

Plaque coverage

Plaque coverage

$\square$ APP/PS1

$\square$ APP/PS1 PB1-KO
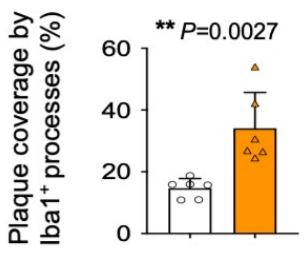

APP/PS1

$\square$ APP/PS1 PB1-KO

E

Peri-plaque glial net

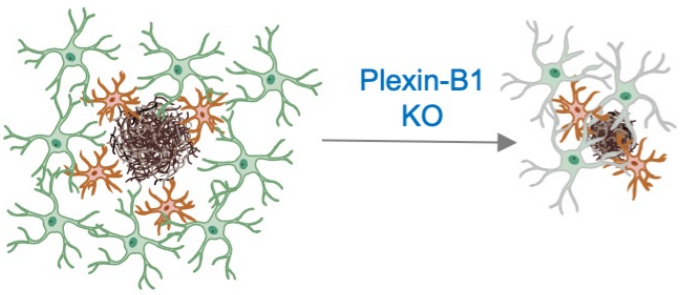

$$
\text { Amyloid plaque }
$$

Figure 2. Plexin-B1 KO results in smaller but more compact peri-plaque glial nets in mouse AD model. 
A) Representative IF images of matching cortical areas show reduced number and size of reactive glial nets surrounding amyloid plaques in APP/PS1 PB1-KO mice as compared APP/PS1 mice. Cortical sections were stained for $\mathrm{A} \beta$ (antibody 6E10), reactive astrocytes (GFAP), and DAPI (nuclear counterstaining). Dashed ovals denote larger glial nets, dashed boxes denote smaller glial nets around compact plaques.

B) Confocal IF images of plaques and glial nets in APP/PS1 and APP/PS1 PB1-KO mice. Dashed lines outline the perimeter of glial net territory defined by $\mathrm{GFAP}^{+}$cells. Quantifications show that Plexin-B1 KO caused reduced glial net sizes and shorter distance of $\mathrm{GFAP}^{+}$cells to plaque margin . Unpaired $t$-test. $\mathrm{n}=9$ sections per genotype, from 3 independent mice each; for glial net size, each data point represents the mean of 11 randomly selected peri-plaque glia nets from one section; for cell distance to plaque, violin plots from $n=104$ and 55 cells for different genotype, showing median, $25-75 \%$ quantiles, and minimal and maximal values.

C) Quantifications indicate lower number and area of $\mathrm{GFAP}^{+}$cells in glial nets in APP/PSI PB1-KO mice. However, the density of $\mathrm{GFAP}^{+}$cells in reactive glia nets and coverage of amyloid plaques by $\mathrm{GFAP}^{+}$glial processes are increased by PB1-KO. Unpaired $t$-test. $\mathrm{n}=9$ sections per genotype from 3 independent mice (each data point represents the mean of randomly selected glia nets from one section).

D) Quantifications indicate lower number, but higher density, and increased area of $\mathrm{Iba}^{+}$microglia in peri-plaque glial nets of $A P P / P S 1 \mathrm{~PB} 1-\mathrm{KO}$ mice. Also note higher coverage of amyloid plaques by $\mathrm{Iba}^{+}$processes in $A P P / P S 1$ PB1-KO condition. Unpaired $t$-test. $\mathrm{n}=9$ sections from 3 independent mice of each genotype (each data point represents the mean of randomly selected glia nets from one section).

E) Schematic depiction of Plexin-B1 knockout phenotypes: smaller but more compact glial nets surrounding amyloid plaques, with fewer reactive astrocytes and activated microglia, reduced cellular spacing (thus higher density), and increased proximity and plaque coverage by glial cells. 
A scRNA-seq: 6 mon prefrontal cortex
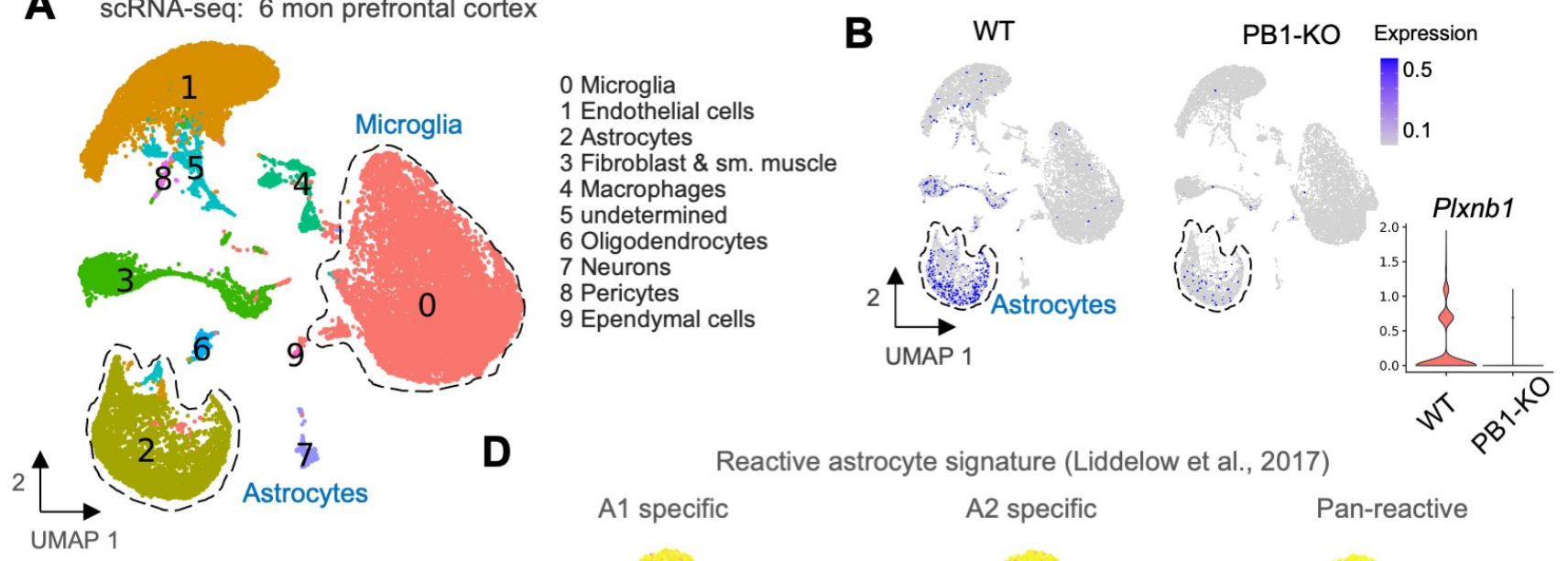

A1 specific

A2 specific

Pan-reactive
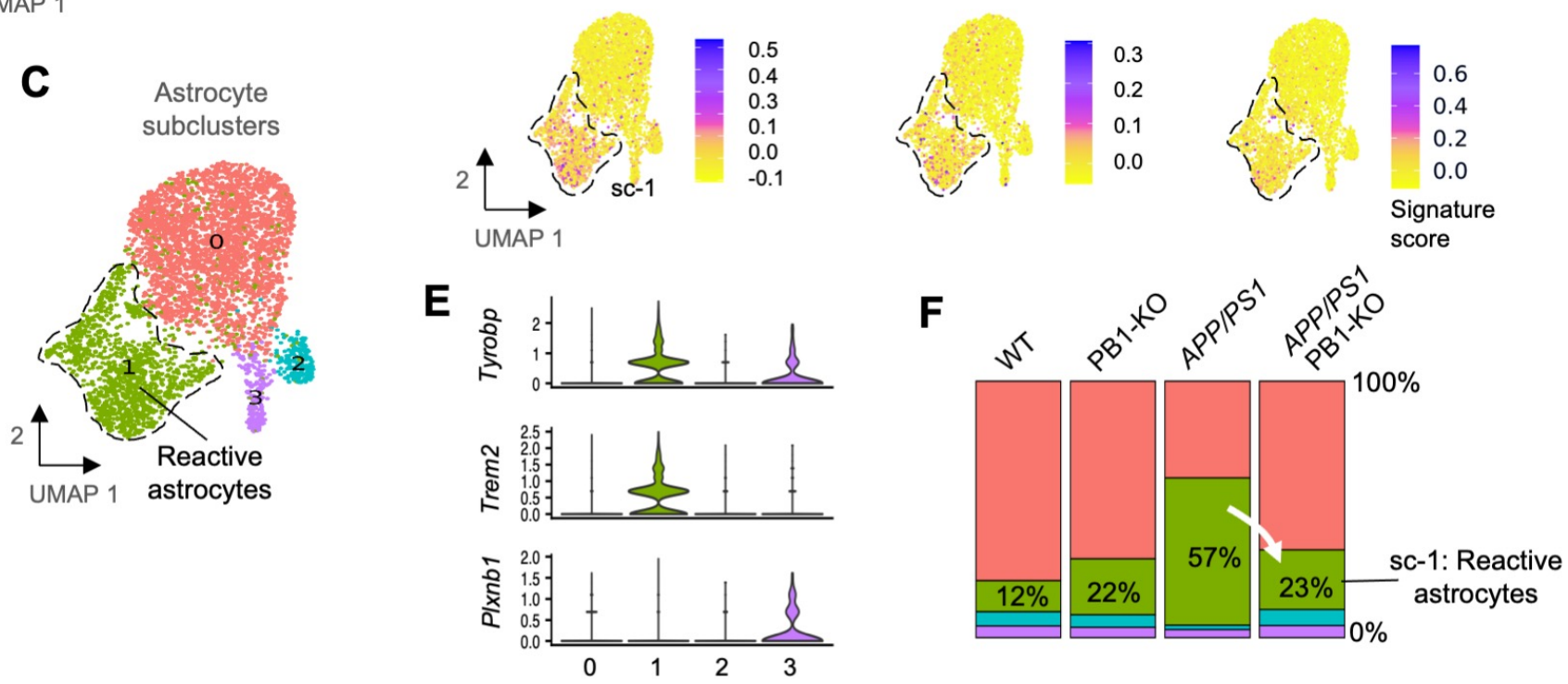

G

Astrocyte sc-1

APP/PS1 PB1-KO vs. APP/PS1

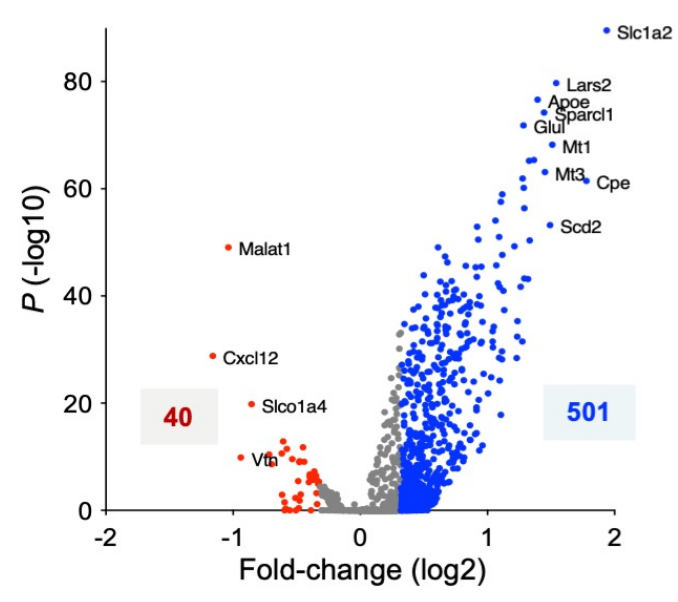

H

Upreg. DEGs in Astrocyte sc-1 in PB1-KO
Negative reg. of amyloid- $\beta$ formation - NTRK2;PRNP;RTN3;APOE;CLU reg. of long-term synaptic potentiation $\begin{aligned} & \text { L-aspartate transmembrane transport } \\ & \text { SLC1A2;SLC1A3 }\end{aligned}$ Glutamate catabolic process Arachidonate-CoA ligase activity Cytochrome-c oxidase activity Ca-independent phospholipase A2 activity Aldehyde-lyase activity Astrocyte projection Mitochondrial resp. chain complex IV

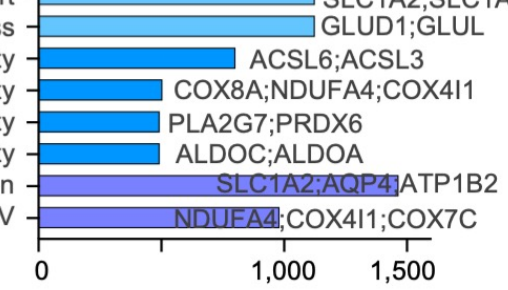

Downreg. DEGs in Astrocyte sc-1 in PB1-KO

Cell junction disassembly
Complement activation, class. pathway
Reg. of TNF superfamily cytokine production
Reg. of glial cell migration
TGF $\beta$ receptor binding

Figure 3. scRNA-seq reveals an impact of Plexin-B1 deletion on the activation state of reactive astrocytes in 


\section{AD brains.}

A) scRNA-seq on prefrontal cortex from 6-month-old mice (WT, PB1-KO, APP/PS1, APP/PS1 PB1-KO) by 10X Genomics system. UMAP embedding of all cells combined show 10 distinct cell type clusters.

B) Feature and violin plots show that Plxnb1 is predominantly expressed in astrocytes, and is effectively ablated in PB1-KO mice.

C) Subclustering of astrocytes identified 4 distinct subclusters, with subcluster sc-1 representing reactive astrocytes.

D) Signature scores plotted on astrocyte UMAP representation for gene sets of reactive astrocytes: A1-specific, A2specific, and Pan-reactive astrocytes (defined in Liddelow et al., 2017).

E) Violin plots illustrate the expression patterns of genes in different astrocyte subclusters.

F) Graphs showing representation of astrocyte subclusters in different genotypes. APP/PS1 caused expansion of subcluster sc-1 reactive astrocytes, which was reduced by PB1-KO.

G) Volcano plot showing differentially expressed genes (DEGs) in astrocyte subcluster 1 between APP/PS1 PB1$\mathrm{KO}$ and $A P P / P S 1$ genotypes. Cut-offs for coloring are 1.25-fold up- or down-regulation, respectively.

H) Enrichment analysis for gene ontologies of DEGs in astrocyte subcluster sc-1 (APP/PS1 PB1-KO vs. APP/PS1). 
A

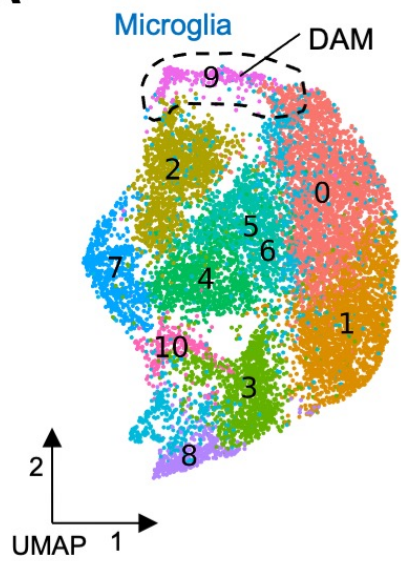

B Disease associated microglia (DAM) marker genes (Keren-Shaul et al., 2017)
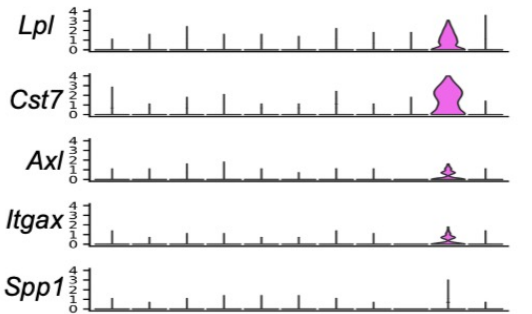

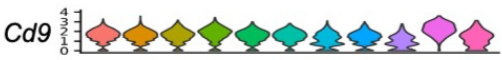
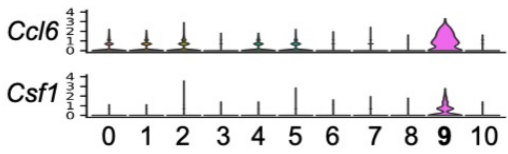

Microglia subclusters
C Homeostatic microglia genes
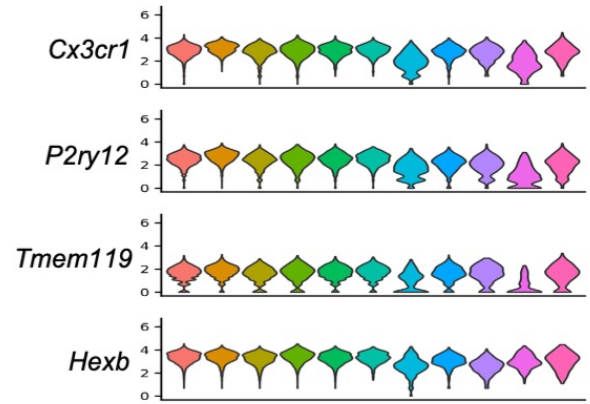

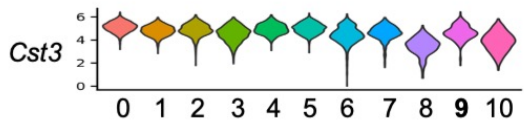
Microglia subclusters

D
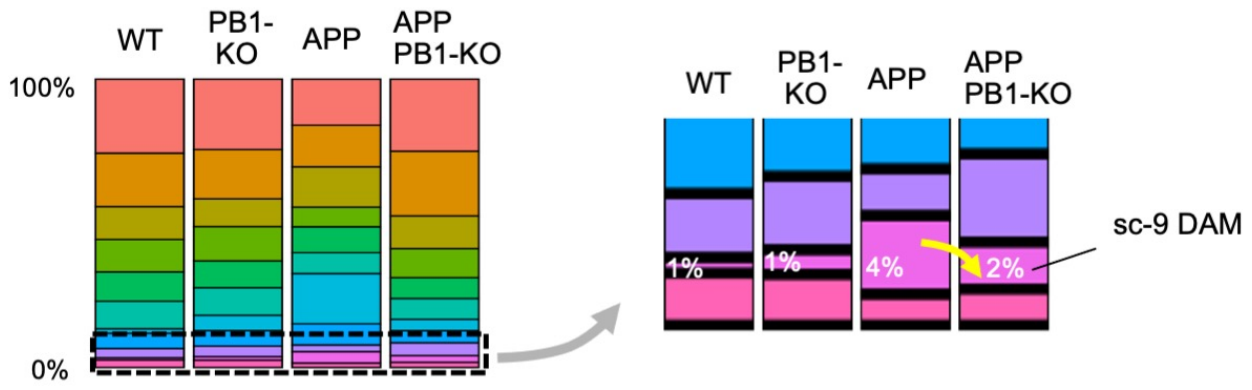

E

sc-9 microglia (DAM) APP/PS1 PB1-KO vs. APP/PS1

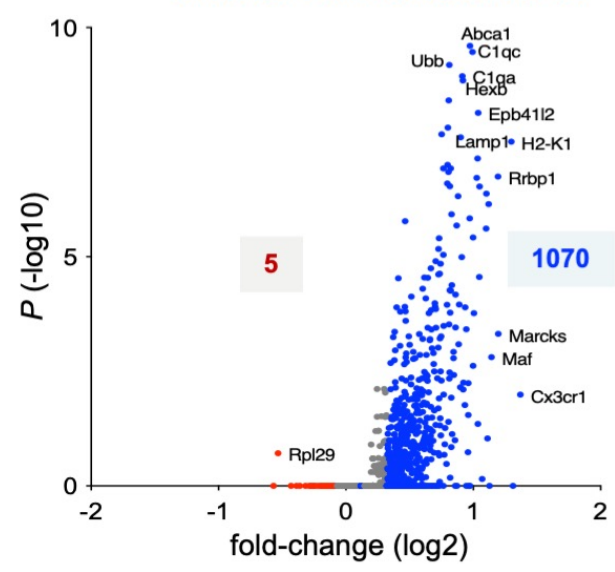

\section{$\mathbf{F}$ \\ Upreg. DEGs in sc-9 microglia (DAM) APP/PS1 PB1-KO vs. APP/PS1}

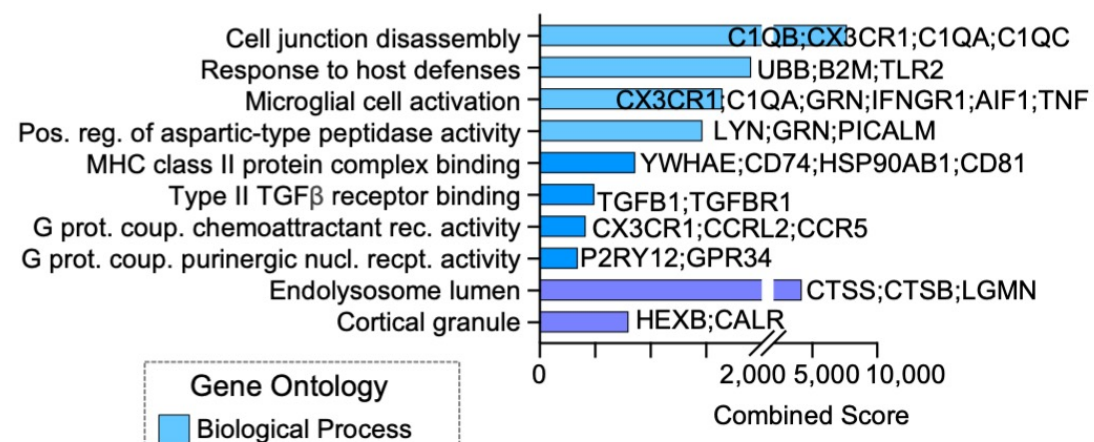

Molecular Function

Cellular Component

Figure 4. scRNA-seq reveals altered microglial activation in APP PB1-KO mice.

A) Subclustering of microglia shown in UMAP embedding, highlighting subcluster 9, which represents diseaseassociated microglia (DAM).

B) Violin plots show the expression of DAM marker genes in microglia subclusters. Note the high expression in microglia sc-9.

C) Violin plots show the expression of genes associated with microglia homeostasis. Note lower expression of 
Cx3crl, P2ry12, and Tmem119 in microglia sc-9.

D) Graphs show altered proportions of microglia subclusters in different genotypes. Note that the microglia sc-9 was expanded in APP/PS1 mice, which was decreased by PB1-KO.

E) Volcano plot showing differentially expressed genes (DEGs) in the DAM-like microglia sc-9 (APP/PS1 PB1-KO vs. APP/PS1). Cut off of 1.25-fold change for coloring. Note that majority of the DEGs were upregulated by PB1KO.

F) Enrichment analysis of upregulated DEGs in microglia sc-9 (APP/PS1 PB1-KO vs. APP/PS1) show enhanced microglial cell activation in APP/PS1 PB1-KO condition. 
A

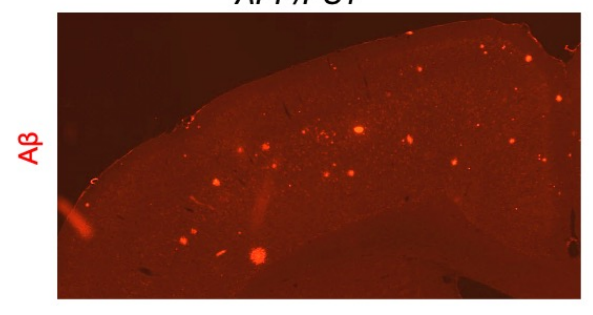

B
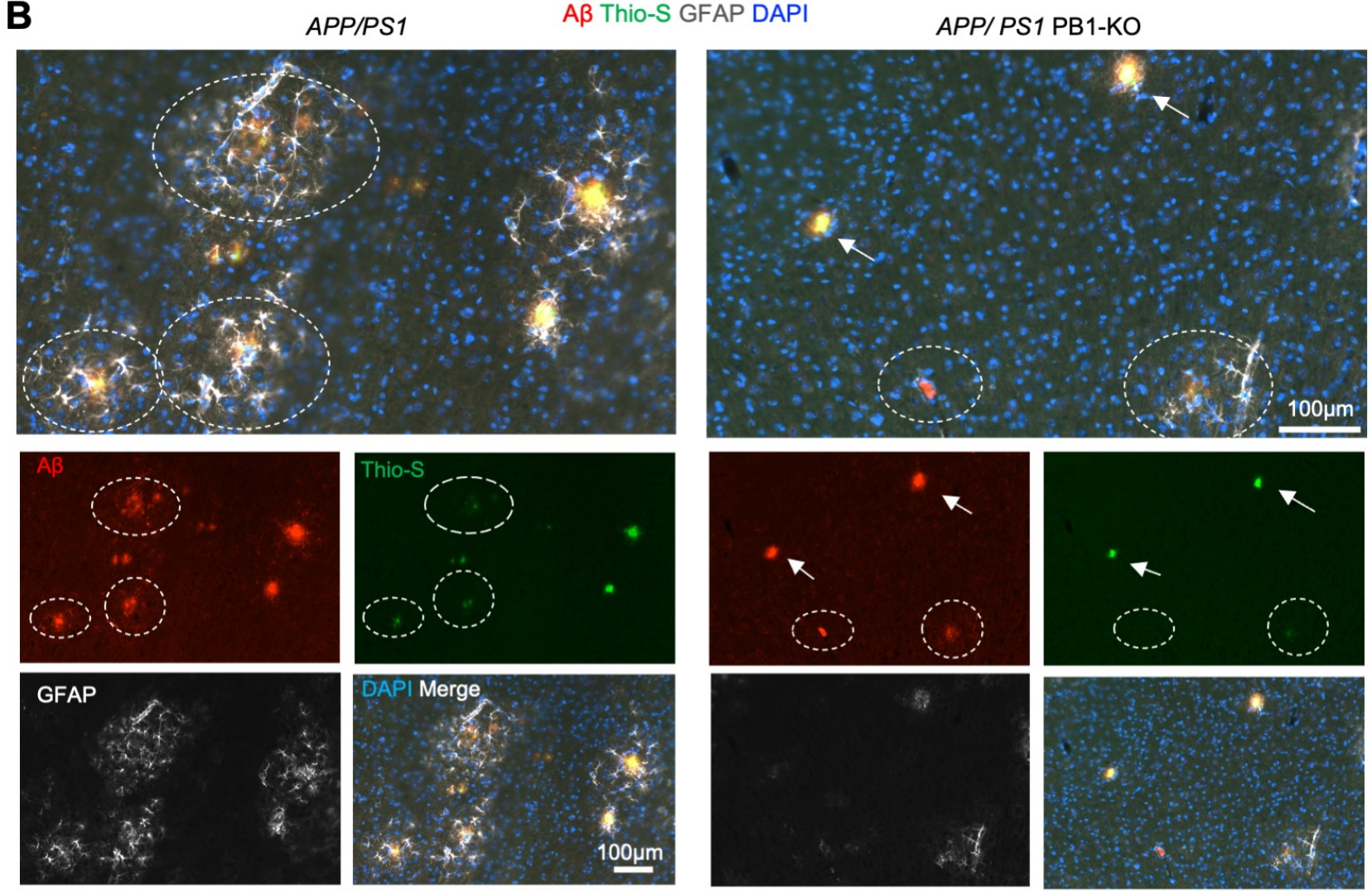

C

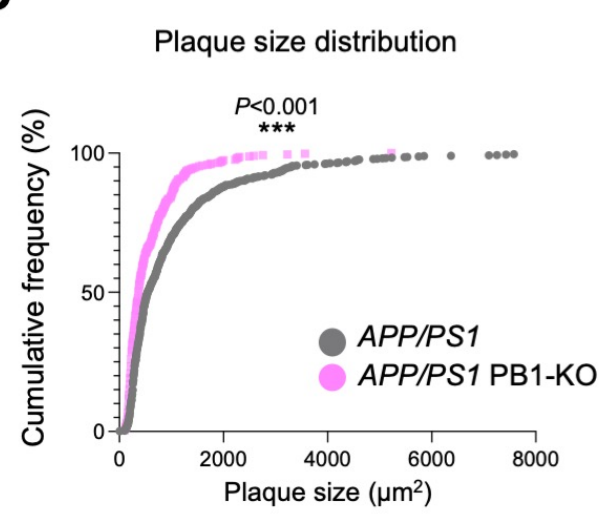

D

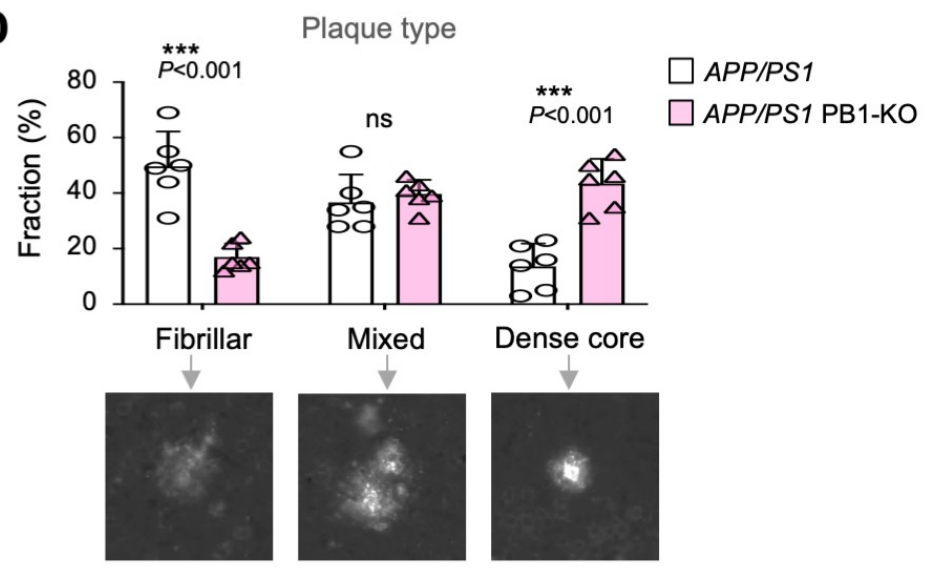

Figure 5. Reduced plaque burden and shift towards dense-core type in AD mice with Plexin-B1 KO.

A) Representative images of forebrain sections from 6-month-old APP/PS1 mice without or with Plexin-B1 KO, stained for amyloid plaques (antibody 6E10). Quantifications show reduced overall numbers of plaques and smaller 
plaque areas in PB1-KO. Unpaired $t$-test. $\mathrm{n}=9$ sections per genotype, from 3 independent mice each. For plaque size, each data point represents the mean of all plaques from one section.

B) IF images of forebrain sections stained with amyloid binding dye Thio-S highlight a shift of plaques towards dense core types (arrows) in APP/PS1 PB1-KO mice. Dashed ovals denote the more diffuse plaques that are only weakly stained by Thio-S. Note also reduced size of glial nets in PB1-KO, as detected by GFAP staining.

C) Quantification of cumulative frequency distribution show that the plaques areas (6E10 antibody staining) are more compact in Plexin-B1 KO mice. Two-way ANOVA with Sidak's multicomparison test. n=741 (APP/PS1) and 428 (APP/PS1 PB1-KO) plaques from 9 sections of three independent mice per genotype.

D) Proportions of plaque types are shifted from fibrillary to compact dense core in Plexin-B1 KO animals. Scoring scheme is illustrated by examples shown below. $\mathrm{n}=6$ brain sections per genotype. Unpaired $t$-test. n.s., not significant. 
A
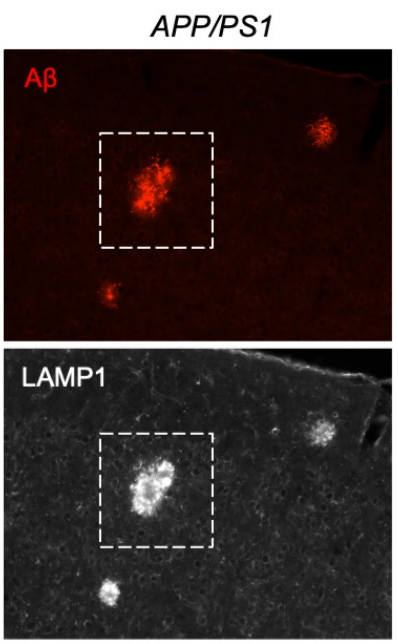

B

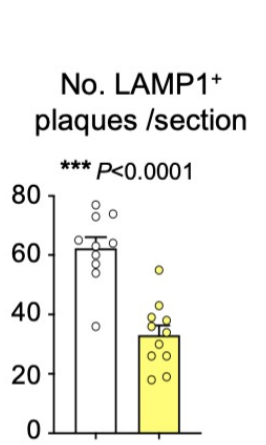

$\square$ APP/PS1
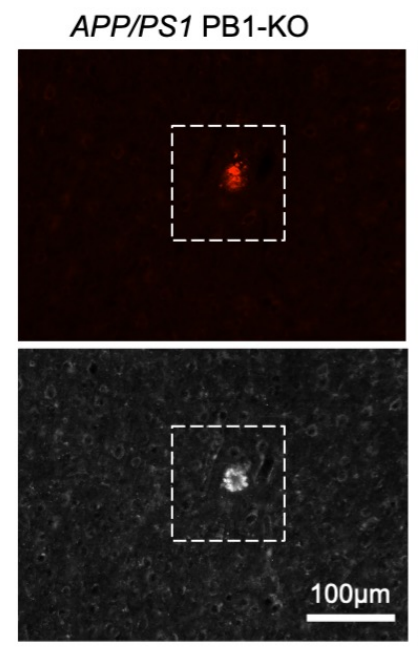

APP/PS1 PB1-KO
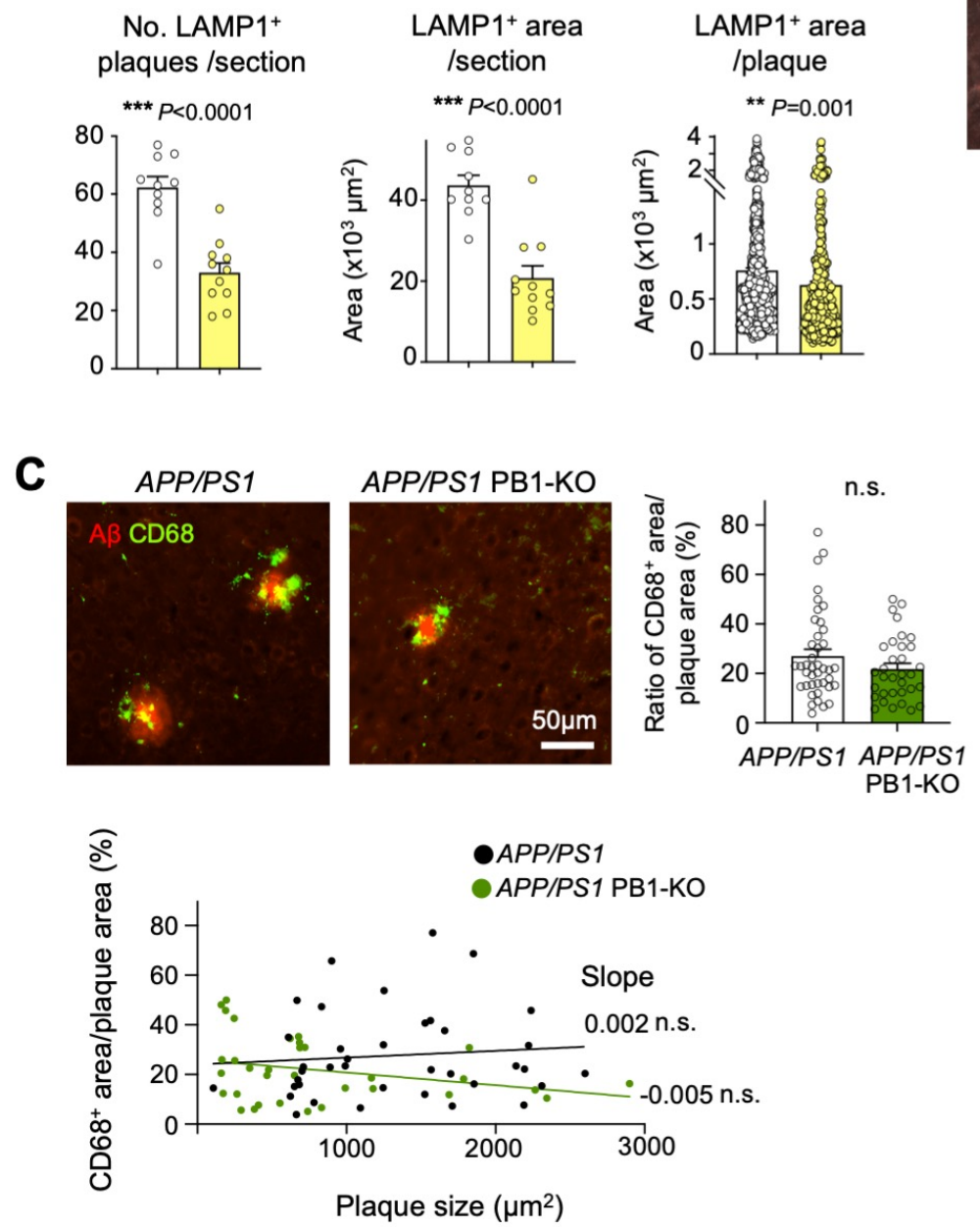

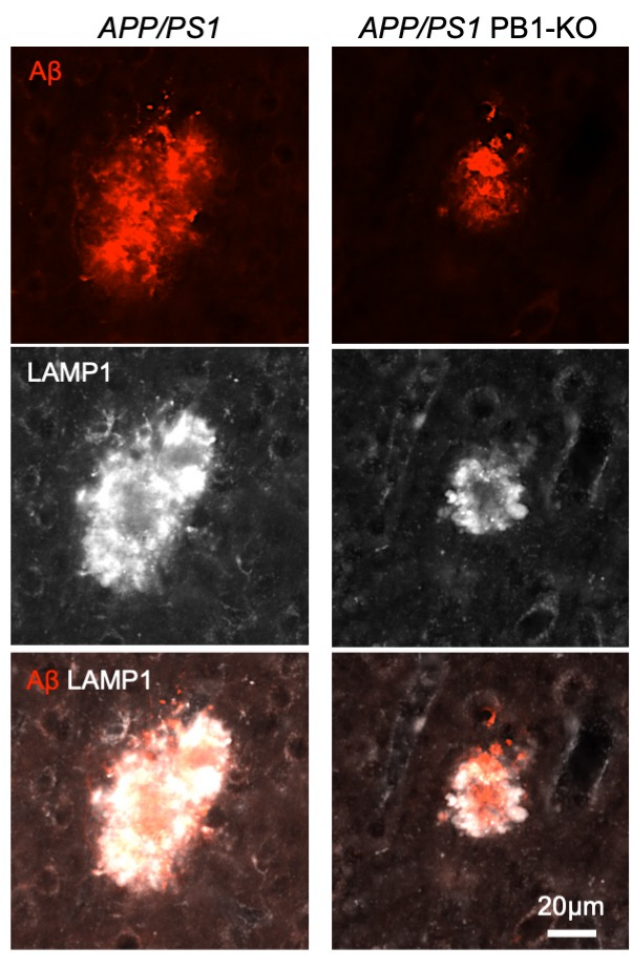

D Reactive glial net surrounding plaque
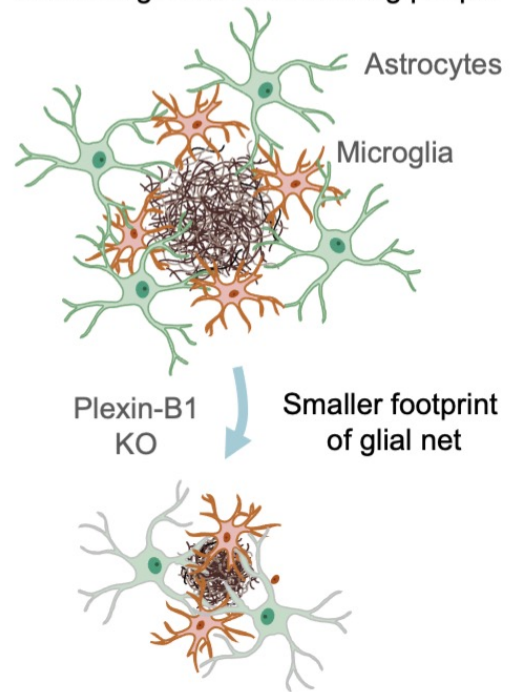

- Relaxed cell distancing in glial net

- Higher plaque coverage by glial processes

- Transcriptomic changes in glial cells

- Lower plaque burden / shift to dense core type

- Reduced neurotoxicity

Figure 6. Decreased neuritic dystrophy in Plexin-B1 KO AD mice.

A) IF images show representative examples of staining for plaques and neuritic lysosomal marker LAMP1. Enlarged images of boxed areas are shown on the right. Note reduced dystrophic neurites (LAMP1 ${ }^{+}$) and more compact plaques 
in 6-months old $A P P / P S 1$ and $A P P / P S 1$ PB1-KO mice.

B) Quantifications show reduced number and area of LAMP1 ${ }^{+}$peri-plaque structures per coronal section of forebrain at level of striatum in Plexin-B1 KO mice. Unpaired $t$ test. $\mathrm{n}=9$ sections from three independent mice per genotype. The size of $\mathrm{LAMP} 1^{+}$area per plaque was also reduced in PB1-KO condition. Unpaired $t$ test. $\mathrm{n}=623(A P P / P S 1)$ or 364 (APP/PS1 PB1-KO) plaques.

C) IF images of representative examples of amyloid plaques surrounded by phagocytic cells labeled for CD68. Quantifications show that the relative area of CD68 in relation to the plaque is comparable between APP/PS1 and APP/PS1 PB1-KO. n=41 (APP/PS1) or 33 (APP/PS1 PB1-KO) plaques, from three independent mice per genotype. For bar graph: Unpaired $t$-test. For dot plot: Simple linear regression test, deviation of slope from zero. n.s., not significant.

D) Graphical summary of a protective effect of Plexin-B1 deletion in AD by reducing the footprint of plaque-related reactive glial nets and reducing neurotoxicity in AD brains. 


\section{Supplementary Files}

This is a list of supplementary files associated with this preprint. Click to download.

- HuangetalSupplementalData.pdf 